OUPT-98-64-P

cond-matt/9808240

\title{
Scaling Laws For the Market Microstructure of the INTERDEALER BROKER MARKETS
}

\author{
DAVID ELIEZER周 \\ Research and Risk Management Dept. \\ General Re Financial Products \\ 630 Fifth Ave., Ste. 450 \\ New York, NY 10111, USA \\ and \\ IAN I. KOGAN"] \\ Theoretical Physics, Department of Physics \\ University of Oxford, 1 Keble Road \\ Oxford, OX1 3NP, UK
}

\begin{abstract}
We discuss a series of simple models for the microstructure of a double auction market without intermediaries. We specialize to those markets, such interdealer broker markets, which are dominated by professional traders, who trade mainly through limit orders, watch markets closely, and move their limit order prices frequently. We model these markets as a set of buyers and a set of sellers, whose numbers vary in time, and who diffuse in price space and interact through an annihilation interaction. We seek to compute the purely statistical effects of the presence of large numbers of traders, as scaling laws on various measures of liquidity, and to this end we allow our model very few parameters.
\end{abstract}

*deliezer@genre.com

†i.kogan1@physics.ox.ac.uk 


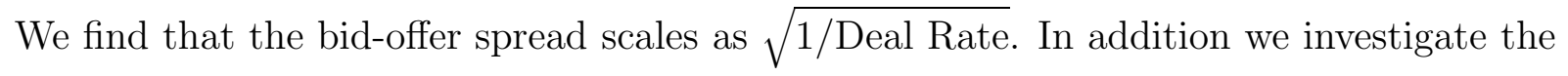
scaling of other intuitive relationships, such as the relation between fluctuations of the best bid/offer and the density of buyers/sellers. We then study this model and its scaling laws under the influence of random disturbances to trader drift, trader volatility, and entrance rate. We also study possible extensions to the model, such as the addition of market order traders, and an interaction that models momentum-type trading. Finally, we discuss how detailed simulations may be carried out to study scaling in all of these settings, and how the models may be tested in actual markets. 


\section{Introduction.}

Market microstructure has made considerable progress in the past few years in understanding price formation in formally organized markets. Most particularly this has been done by considering the dealer's optimization problem. Starting with the work of Garman [3], and continuing with Stoll [4], Ho and Stoll [5], O'Hara and Oldfield [6], and Amihud and Mendelson [7], the various elements of this problem and its consequences have gradually been elucidated 3 , 4, 5, 6, 0, 8, 9, 10, 11, 12, 13, 14, 15, 16, 17, 18, 19, 20, 21, 22, 23, 24, 25, 26]. For a more complete bibliography, see [1, 2].

A separate but parallel line of research, also beginning with Garman, and extending through Cohen-Maier-Schwartz-Whitcomb [8, 11], has considered the structure of double auction markets, markets without intermediaries. Such markets are simpler to treat than the dealer problem. This is because the large number of traders are considered to act independently of one another and their aggregate behavior may therefore be treated using well developed stochastic methods, as a purely statistical system. Thus it is considered that the great press of numbers, not any complex social interaction, or difficult optimization problem, is the principle determinant of large scale market behavior in a double auction market. In this regard Garman [3] first studied trade price distributions over time in a double auction market. In [8] Cohen, Maier, Schwartz, and Whitcomb considered the time to trade in such a market, under the assumption of finite-sized trader movements. In [11], they studied the effect of "thinness" on market returns, where thinness, the value of shares outstanding, was used as a proxy for liquidity.

More recently, price evolution in double auction markets was studied by Bak, Paczuski and Shubik (BPS) [27], who introduced a model in the same vein as the Garman model, with additional features that effectively mapped double auction market dynamics onto a type of model from chemistry, known as diffusion-controlled annihilation. Like Garman, they used this to study trade price distributions over time, obtaining a model that goes beyond the standard Brownian motion model, to recover the empirically observed "fat tail" distributions, and the associated Hurst exponent. The diffusion-controlled annihilation model relevant to markets is a model in one dimensional space, with initially segregated reactants, and forms an interface, a "reaction front". This front was first studied by Gálfi and Rácz 28, and then later was developed in a series of papers by Cardy and coworkers 29], and studied numerically by several groups [30. Early contributors to the theory of diffusion-controlled annihilation include Doi [31], and Peliti[32], and a good 
review is contained in [33] ( for a general introduction into kinetics of diffusion controlled processes, see, for example, book [34]).

In the Garman model traders enter the market at a given price, and if their trade price is not hit, they leave the market a certain time later - they do not change their trade price while they have an open interest. This is a behavior characteristic of "investors", traders who buy and sell rarely, and hold for long periods of time, for whom trading tactics to obtain the optimal trade price are not crucial. Many markets are in fact dominated by trading of this kind. However, there are also markets which are dominated by professional traders, who typically work for a sell side institution, watch the markets every minute, and care very much about saving every basis point. The best examples this kind of market are the interdealer broker markets, which exist for swaps, caps, floors, and the treasury market, to name a few. This kind of trader places mainly limit orders in the market, and may change his or her limit order price many times before trading, based on how he or she perceives the direction of the market, the direction of related markets, changing hedging needs, and anticipation of news bulletins. An external observer might characterize this behavior as a random walking behavior, and that the collection of all such traders (i.e. their limit order prices) in this market would then be seen to diffuse. This behavior stands in contrast to those studied empirically by [26]. We wish in particular to describe the microstructure of this type of market. We therefore shall study a model in which traders both diffuse and annihilate. We shall then see that the diffusion property of the model has strong and fascinating implications for microstructural behavior.

In this paper, like that of Garman and BPS, we shall study a double auction market as a statistical system. Rather than studying the distribution of trade prices, however, we focus on using this model to calculate scaling laws for expectations of the various quantities that describe microstructural dynamics, e.g. the bid-offer spread, time-to-midmarket trade, bid or offer size (or more generally, the density of traders), and deal rate. Taken together, these four quantities describe, somewhat redundantly, the notion of liquidity. Although liquidity itself has no unique quantitative definition, each of these quantities is monotonic in liquidity, and we may define liquidity scaling laws as the functional behavior of each of these in terms of the others. In this paper we shall consider liquidity scaling laws as a function of the deal rate. Existing intuitions then govern the qualitative behavior of these laws as follows.

The higher the deal rate, the smaller the bid-offer spread.

The higher the deal rate, the more quickly we can trade at the midmarket. 
The higher the deal rate, the greater the density of traders.

The quantities one encounters in studying market microstructure are measured over short time scales, minutes and seconds. This feature simplifies empirical studies, but more importantly, we argue, it simplifies the nature of the model itself. This is because the short time scales leave little or no time for communication between traders. This implies that we do not need to model the complicated sociological interactions that take place when large amounts of information passes between traders - only the great press of

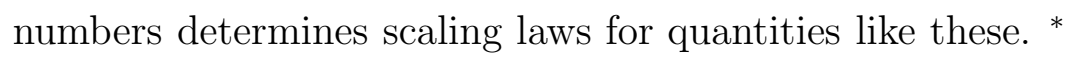

Making all these intuitions into quantitative scaling laws is the principal aim of this paper, and these present a set of new and interesting research problems. In addition to the scaling laws associated with various measures of liquidity, there are large sets of other interesting problems regarding internal market structure over short time scales. In this paper, we will also investigate scaling laws concerned with details of steady state markets, market making strategies, and a market's response to imbalances between buyers and sellers. We note here that scaling laws have been used before to characterize the behavior of markets - the best known example is the Hurst exponent. However, this example serves to highlight the difference between our work and previous work, especially [27 whereas scaling laws such as the Hurst exponent quantify the behavior of price evolution in time, and so are scaling laws in the time variable, ours are concerned with issues of price formation, and are therefore described by scaling laws with respect to liquidity.

In the next section we will demonstrate how this can be done. We shall first introduce a mathematical framework, statistical field theory, which allows us to treat systems, such as a market, with variable numbers of random agents, similar in this way to Garman's model. Then we will seek a model which is minimal, i.e. the simplest model possible which describes the market phenomena mentioned above. We will see how these considerations define this model almost uniquely, and will find the model to be similar to the dynamics of the "annihilation" reaction $B+S \rightarrow \emptyset$ in a one-dimensional space, a well studied problem (for further reading see the excellent recent review [33]). While Garman [3] was the first

* We distinguish "sociological" interactions, the collective interaction of traders with each other, from "psychological" interactions, the response of individual traders to changes they perceive in the market. While sociological interactions ought not be important over these very short time scales, psychological interactions might, if they were to act in a correlated fashion. Our approach carries with it an implicit but important assertion, that these interactions are in fact uncorrelated over short time scales, and therefore "wash out", so that we may treat their behavior as a collection of random walks. 
to introduce the analogy to this type of reaction into finance, an important element later added to this picture was the idea of diffusion. Bak, Paczuski, and Shubik, [27] were the first to introduce to finance a model with both diffusion and this annihilation reaction, in a study of price evolution. Interestingly, it is the one-dimensionality of the system (a consequence of the standardization of the trading contract) that gives this model nontrivial dynamics. In the next section we will present a more complete argument for this mapping of our problem onto this model, define some financially relevant variables, and use this to show explicitly how the above scaling laws, and many more, may be calculated within such a model.

This minimal model is very easily treated, and there already exists a considerable body of work devoted to it in the physics and chemistry literature [33. When we apply this model to market microstructure, we shall find that several of the scaling laws and correlations we seek are calculable by known analytic methods. This minimal model is intended to describe market statistics at only the level appropriate to scaling laws, and to this end we keep the number of parameters to a minimum. In addition to the deal rate, we shall require only two other parameters to define the model completely - these turn out to be the volatility (or diffusion coefficient) of individual traders, and the effective width of the price space. By calculating and measuring any of the correlation functions these parameters are easily inferred.

\section{Going Beyond the Minimal Model}

The notion of studying the scaling laws of a market's internal structure may be extended beyond markets in the normal state. In this paper we shall also introduce models which explore scaling laws in other settings. With the exception of the two-fluid model in its crashing phase, the additional effects of these models are weak, and modify the dominant effect of statistics only by small corrections.

\section{- The Effect of Random Disturbances}

The minimal model with which we begin this study is intended to model "quiescent" markets. i.e. those in which deals are steadily ticking over at a constant rate, and in which no catastrophes are occurring. We may extend ourselves beyond this most ideal regime into others, by first considering the effects of random disturbances within the market. We can do this by imagining that they cause trader dynamics, defined by their drift, volatility or flux, to jump around randomly. The strength of this random noise component of these quantities behaves somewhat like a temperature, i.e. the amount of "energy" injected 
into the market by the influence randomly occuring series of external events. We imagine that the market is continually buffeted by a series of small jolts, that force it partially into its excited states. The strength of this noise controls the weighting of the system in these states, and introduces noise-strength dependent corrections to the correlations of market observables.

\section{- Scaling Laws in the Crashing Phase: the Two Fluid Model}

A market which is in the process of a long crash does not look quiescent. However, it is possible to regard a market in free-fall as a market in a different phase, subject to a different sort of dynamics, but one which may be probed by the same sort of statistical scaling laws nevertheless. It is not sufficient, for this purpose, to simply consider the minimal model in the presence of a large imbalance between buyers and sellers, because this would not recover the well known phenomenon of the widening of the bid-offer spread. We believe that the missing element, ignored by the minimal model, is market order traders. The minimal model contains only traders who enter limit orders into the system, so that the true best bid/offer can be seen on screen, but this would not represent the reality of a crash, during which most traders attempt to hit the best bid directly, as a market order. To address this, we introduce a "Two-Fluid Model", one in which there are two flavors of buyer, $B$ (limit order bidders)and $B^{\prime}$ (market order bidders), and two flavors of seller $S$ (limit order sellers) and $S^{\prime}$ (market order sellers). The prices of the unprimed traders only are used to calculate the best bid and offer on the trade screen, and are therefore visible to other traders. Thus the Two-Fluid Model includes the reactions $B+S \longrightarrow 0, B^{\prime}+S \longrightarrow 0$, and $B+S^{\prime} \longrightarrow 0$, but not $B^{\prime}+S^{\prime} \longrightarrow 0$, because these primed traders are invisible to each other. This model, when the population of invisible $S^{\prime}$ traders is large, now has the familiar behavior in which the bid-offer spread widens out during a crash. We pause here to note that Bak, Paczuski, and Shubik introduced "heterogeneous" models, too, ie. models involving more than one types of trader [27].

- The onset of crashes: the Bias Model

We have argued that the short time scales of our measurement enable us to ignore collective, sociological effects of trader behavior, because they will not have had time to communicate appreciably. But, if we were to consider slightly longer time scales, it would be easy to identify the most direct and likely medium for this communication - it is the trading screen itself, which instantly informs traders of trade prices. Over slightly longer time scales, it also allows them to observe trends. We may get more ambitious and attempt to go beyond the very shortest time scales, to model the most immediate of 
collective behavior. We treat an interesting model of this type in this paper, as well.

We introduce a simple model that treats trend-following behavior in traders under the name of the "Bias Model". The rate at which trend-following or "bias" builds up in this model is controlled by an external parameter, which we call the "market tension", a property of the market which we will measure, and which we expect to be slowly varying. When this trend-following parameter is positive, the traders' drift is increased each time the trade price increases, and decreased each time the trade price decreases - this is meant to correspond to momentum-trading. When bias is negative, so that the traders' drift moves in the direction opposite to the trade price, this corresponds to bargain-hunting/profit-taking.

With positive tension, this model is intended to develop instabilities which mimic market crashes, and preliminary simulations confirm that this is so. The model builds up bias slowly at first and, depending on the market tension parameter and the starting point of the trader drift, the market eventually builds up enough drift to leave the diffusiondominated regime. At this point it goes into a ballistic regime, in which drift plays a significant or dominant role - the crashing phase. We propose to use this model to study scaling laws in market microstructure in the prelude to a crash.

- The Diffusion Proposal and the Dealer's Optimization Problem: A modification of the order arrival process

Although it is outside the main development, we shall also use our dynamical framework to consider a problem in market making. We ask the question: what is the optimal bid-offer spread that a monopolist market maker can set, as a function of time, given that he has a view of all of the limit orders placed in the market? This dealer optimization problem has been considered in great detail by Garman [3], Stoll[国],Ho and Stoll[5], O'Hara and Oldfield [6], among many others. We do not wish in this paper to engage this much more complicated topic - our intent is simply to suggest that the Poisson process model for order arrival may be refined by using the known initial data of limit orders and the diffusion proposal, to obtain a sharper estimate of order arrival. We introduce a simple model for maximizing profit while minimizing the net open position, which illustrates this method.

\section{Testing the Models}

In the last section of this paper we shall discuss the testing of the predictions of these models. We have consciously kept the number of parameters describing the model to a 
minimum, so as to isolate the effect of large numbers of traders and to make the model easily testable. Our testing will be done in a future publication, and will concern markets with institutional traders, and measurements will be made over very short time scales (minutes and seconds), in a single market [35]. In this section we review the previous studies of the spread (principally in dealer markets, which dominated by the long term investors, not the short term traders we are interested in). We also lay out the program for testing experimentally the scalings we have computed, and will compute, for these models.

\section{A Brief History of Physical Approaches to the Financial Markets}

The application to financial markets of analogies to physical systems have a long history starting from Louis Bachelier [36], who first proposed the random-walk model of the stock market. In the 1960s and 1970s these ideas became very popular and eventually lead to the famous Black-Sholes option pricing formula [37] and to Mandelbrot's applications of scaling behaviour to financial markets 38. Recently, a lot of papers have appeared in which markets were treated as far-from-equilibrium dynamical systems [39]. It is impossible here to review all relevant recent papers and areas of research of the quickly growing field of econophysics 40. However, we pause here to mention several notable examples, such as work on scaling behaviour for exchange rates 41], "log-periodic" oscillations as crash precursors [42], dynamics of the interest rate curve [43], and market fluctuations [44, 27]. There are, of course many other deserving papers which we do not have the space to include.

\section{Development of the minimal model}

In this section, we present an argument for the mapping of microstructure of the interdealer broker markets onto a statistical field theory, in which particles diffuse and annihilate, a well known model of chemical reactions.

Configuration Space of the Model

What is the statistical system suggested by our arguments above? Because we seek to compute only the most dominant behavior as a function of liquidity, without the fine detail, we construct first of all the simplest possible model which is consistent with the proposal described in the introduction. 


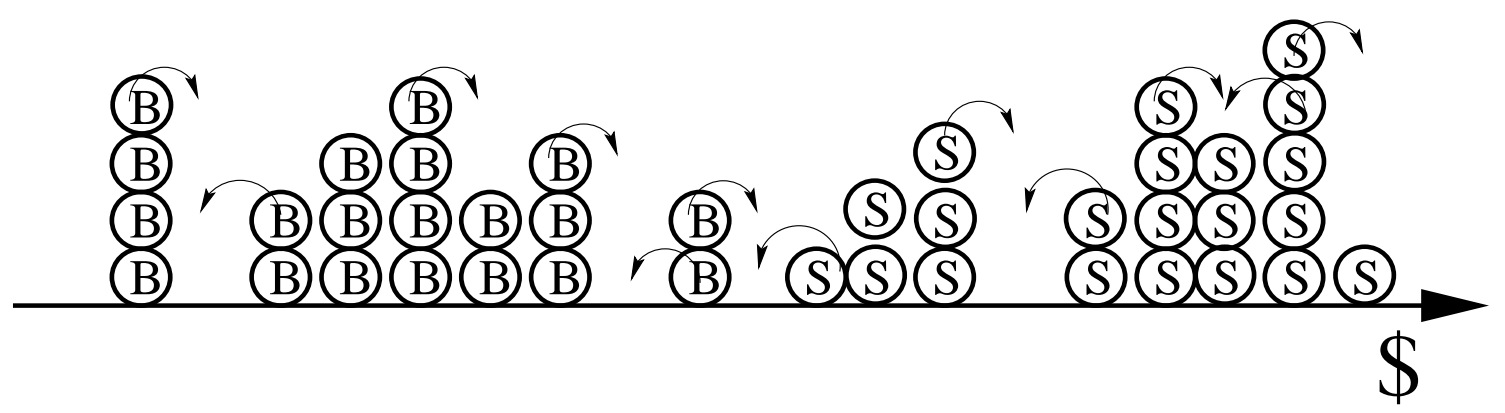

Figure 1: A typical distribution of buyers (balls with letter B) and sellers (balls with letter S). Arrows describe "hops" - not all buyers and sellers will hop in each instant of time.

Within a simple market, we wish to investigate the scaling of the market observables i.e. the best bid, best offer, trade price, deal rate and other things we might infer from a trading screen. In particular, we wish to study their dependence on the number of traders (buyers and sellers) present in the market. This requires us to treat the dynamics of the market observables as functions of the underlying dynamics of individual traders. We do this as follows. To each buyer we associate a price, his bid price, and to each seller his offered price, and imagine the two types of trade prices moving around on a 1 dimensional space we call the "price space" $\mathcal{P}$. (See Figure 1) (Of course, we start our model off by placing all seller prices above all buyer prices. ) It is convenient to regard this space as a discrete one-dimensional lattice on which traders (i.e. their trade prices) "hop" from site to site. In this model each trader trades in a single standard size *

In this setting, each of our market observables is calculable. We shall compute a statistical average of the market observables, and this average will run over the possible positions of all the traders, over a suitable probability measure, specified at a time $t$. This probability measure we refer to as the "state" of our system.

Dynamics of the Model

We wish to study not only the static behavior of the best bid or offer, but the dynamics as well, so we must specify a dynamic law for the movements of the traders. This law is

* This is not a terribly strong restriction, because interdealer broker markets often have a standard size in which much of their trading is done, and are often willing to break up larger trades if necessary 


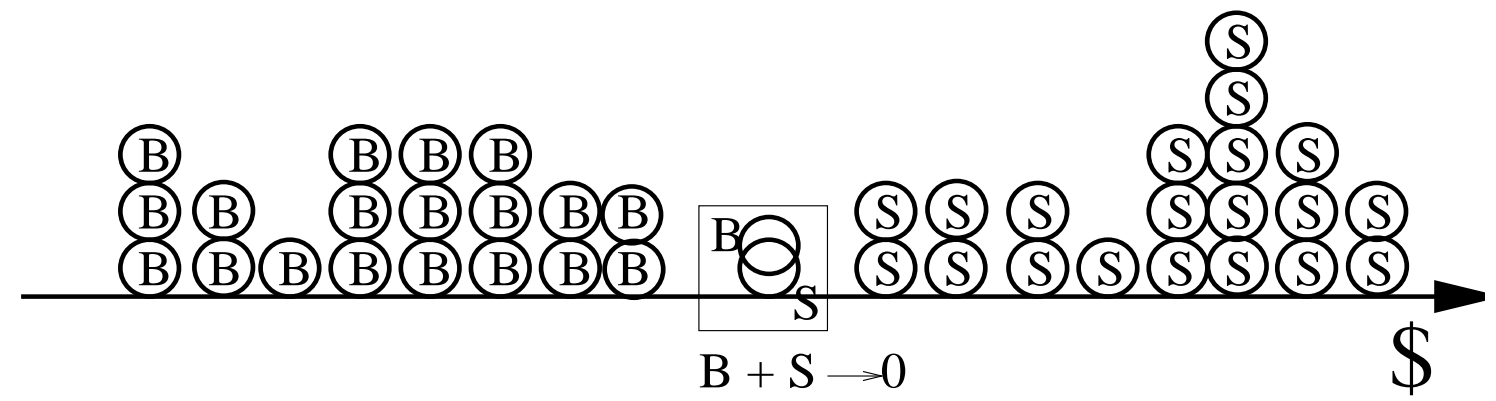

Figure 2: Annihilation $B+S \rightarrow 0$

not completely arbitrary. Any dynamic law we may specify must guarantee that when a buyer and a seller are at the same point in price space, they do a deal and disappear from the market. At each moment in time, therefore, the dynamical law deletes from the state any pair of buyers and sellers it finds occupying the same point in price space. We call this part of the dynamics "annihilation" (See Figures 2 and 3).

As discussed in the introduction, an essential ingredient of our model is that traders in the interdealer markets change their trade prices often while they have an open interest, in an effectively random, uncorrelated fashion and this leads us generally toward a random walk behavior for individual traders, and diffusion for the behavior of the aggregate. The precise nature of this random behavior in price space is somewhat hard to know, and depends on a trader's psychology, etc. We do not wish to attempt to treat the details of these effects, only the effect of the weight of numbers of traders present, an effect which any more detailed theory would also contain. Our model in this sense should be considered "minimal". In keeping with the modest goal of capturing the gross, purely statistical features of random-walking traders only, we introduce the simplest random walk behavior, with zero drift and constant volatility for the traders (hereinafter known as $D$, the trader diffusion constant, or trader volatility). Hence, our minimal model for a trader's price evolution process will consist of diffusion interspersed with annihilation, i.e. it is described by a diffusion controlled (or diffusion driven) annihilation reaction. In the physics and chemistry literature this sort of dynamics has been studied extensively, and a good introduction is contained in [34], and references therein, as well as [33]. In addition, the mapping of market dynamics onto diffusion-controlled annihilation was first used by [27] to study the random process governing trade price evolution. 


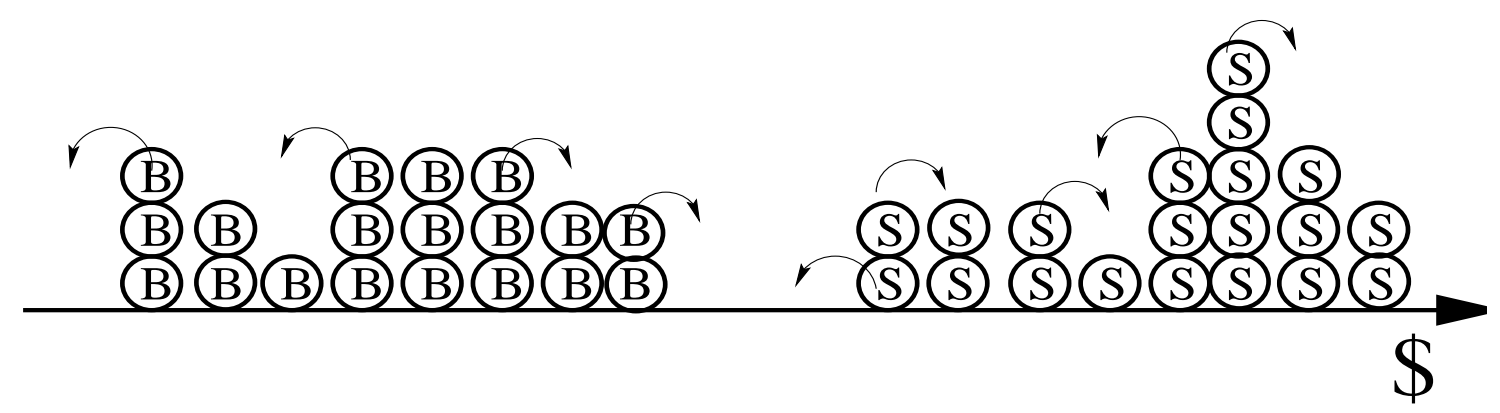

Figure 3: A new distribution of buyers (balls with letter B) and sellers (balls with letter S) after an annihilation (trade) has occured. Note that the bid-offer spread fluctuates. Here it is bigger than in Figure 1

\section{Distribution of Traders}

To compute the scaling laws introduced in the previous section, we must impose a probability measure, the state measure, on the positions of each type of trader. Although we have specified a dynamical law for the state measure, we haven't yet defined it until we have also specified its initial conditions. This represents a lot of missing information, which cannot be deduced from the conditions we have so far imposed on our model - we must discover what kind of diffusion-annihilation dynamics is relevant to our problem. We do this as follows. It is natural, for this kind of problem, to restrict our attention to quiescent markets, i.e. those where catastrophes such as market crashes or large, explosive jumps due to the injection of critical new information are not happening 1 . We assert here that a quiescent market is an approximately steady state market, and thus we define our evolving state measure, at any time $t$, to be that probability measure which is the steady state solution of our diffusion-annihilation dynamics.

As a first approximation, we allow traders to enter the market only at the ends, as though buyers began by bidding very low, and sellers by offering very high, and then improving. This is clearly an approximation which we expect will not change the dynamics too greatly. In a series of numerical simulations we will address the question of more realistic insertion processes, in a future publication 45 .

\footnotetext{
${ }^{\dagger}$ Because our time scales are so short, there should be many such intervals during the day in most markets. Indeed, most of the time, by this definition, most markets are "quiescent".
} 
Like most such models we may solve this minimal model by simulation. However, we can go much further for this model, because it happens to be similar to many well-known analytically soluble models [46], see also [33] and may itself be soluble - in addition, there are approximate analytical methods available [29]. Through these, the set of steady state solutions is well understood, and it is interesting and helpful to note that the set of steady state solutions corresponds to the lowest energy states ("ground states") of the equivalent physical system, and is a family indexed by a single parameter, the deal rate $J$. Thus, given a deal rate, i.e. a market at a given level of liquidity, there is a unique steady state solution to our model. The scaling laws in question may be evaluated simply as an expectation value of the market observable in any such ground state, regarded as a function of the choice of the ground state. Making use of this, many numerical results for scalings have already been found, see for example [30.

\section{The dynamical framework: statistical field theory}

\section{The State is an Evolving Measure on Sequences on the Price Space}

In this section, we introduce the mathematics of our dynamical framework. It is a variant of statistical field theory, first used by Doi [31] and Peliti [32], and it is similar to the approach taken by Garman in his double auction model [3] and Bak-PaczuskiShubik 27]. The scaling laws we seek are moments of the distribution of buyers and sellers at a slice of time $t$. Therefore, we do not need the full distribution on path space, but just a time-slice of this distribution. In summary, we require a distribution on the positions of traders at a time $t$, which evolves in time according to diffusion-annihilation dynamics. It is this distribution, hereafter known as the "state measure" or just the "state", which shall be the dynamical variable of our model.

The measure space underlying this system is different from those one usually encounters in financial problems. Stochastic problems in finance are usually expressed as stochastic differential equations, which lead to a distribution function on an underlying sample space, say $\mathcal{R}^{N}$, of fixed dimension.

If $N$ traders obeyed a simple diffusion law, instead of diffusion-annihilation dynamics, we would describe the system with a set of coupled stochastic differential equations. Solving this system would give us an evolving state measure, $\mu \in \mathcal{M}_{N}$, a measure on the $N$-dimensional underlying sample space $\left(\mathcal{R}^{N}\right.$, in this case). In our problem, however, the dimension of the underlying sample space itself can change during the evolution, because traders may exit or enter the system. The measure space in which there are exactly $N_{T}$ 
traders (i.e. "fixed trader number") is but a small part of the full measure space of our system. In fact, we would like our model to allow the possibility that the system may, along one branch, have had a buyer and seller collide, annihilate, and remove each other from the system, and along the other branch that they did not meet, and therefore still remain within our system, in other words, that the system resides partly in one trader number subspace $\mathcal{M}_{n_{b}, n_{s}}$ and partly in another, $\mathcal{M}_{n_{b}-1, n_{s}-1}$. Here let $\mathcal{P}_{n_{b}, n_{s}}$ be an $n_{b} \cdot n_{s}$ dimensional underlying sample space in which $n_{b}$ buyers and $n_{s}$ sellers are present - then $\mathcal{P}_{n_{b}, n_{s}}$ is a small part of the full underlying sample space $\mathcal{P}_{\text {Full }}=\cup_{n_{b}, n_{s}} \mathcal{P}_{n_{b}, n_{s}} . \mathcal{M}_{n_{b}, n_{s}}$ is the space of measures on $\mathcal{P}_{n_{b}, n_{s}}$, and $\mathcal{M}_{\text {Full }}$ is the space of measures on $\mathcal{P}_{\text {Full }}$. Then we are required to include in our full measure space linear combinations of distributions on all the different underlying sample spaces $\mathcal{P}_{n_{b}, n_{s}}: \mu=\sum_{n_{b}, n_{s}} c_{n_{b}, n_{s}} \mu_{n_{b}, n_{s}}, \mu \in \mathcal{M}, \mu_{n_{b}, n_{s}} \in \mathcal{M}_{n_{b}, n_{s}}$ (where $\mathcal{M}_{n_{b}, n_{s}}$ is the space of measures on the underlying sample space $\mathcal{P}_{n_{b}, n_{s}}$ ), and therefore the full measure space for our stochastic system $\mathcal{M}_{\text {Full }}$ is a vector space direct sum of the measure spaces with fixed trader number $\mathcal{M}_{\text {Full }}=\oplus_{n_{b}, n_{s}} \mathcal{M}_{n_{b}, n_{s}}$. $\mathcal{M}_{\text {Full }}$ is the space of measures on the full underlying sample space $\mathcal{P}_{\text {Full }}$. The norm of the projection into each subspace $\mathcal{M}_{n_{b}, n_{s}}$ is the probability of having $n_{b}$ buyers and $n_{s}$ sellers. As these probabilities must add, the norm on $\mathcal{M}$ is the sum of the fixed subspace norms $\|\mu\|=\sum_{n_{b}, n_{s}} c_{n_{b}, n_{s}}\left\|\mu_{n_{b}, n_{s}}\right\|, \mu \in \mathcal{M}, \mu_{n_{b}, n_{s}} \in \mathcal{M}_{n_{b}, n_{s}}$.

This dynamical system differs in one further respect from systems we would describe with stochastic differential equations. The identity of traders does not matter to us - if buyer 1 sits at site 1 and buyer 2 sits at site 2 , that is the same, for us, as if buyer 1 sat at site 2 and buyer sat at site 1 . As it stands, the system keeps track of more information than we are concerned with. We therefore identify these different configurations as the same point in our underlying space, by modding out by the action of the permutation groups (separately for buyers and sellers) on that space, i.e. $\mathcal{P}_{n_{b}, n_{s}}=\left((\mathcal{P})^{n_{b}} / S^{n_{b}}\right) \otimes\left((\mathcal{P})^{n_{s}} / S^{n_{s}}\right)$. Modding out $\mathcal{P}^{n}$ by $S^{n}$ is the same as restricting the measure space on $\mathcal{P}_{n}$ to symmetric measures on $\mathcal{P}^{n}$ and, to compensate for multiple counting of configurations, adjusting the measure by an appropriate multinomial factor $\mathrm{A}$.

A point in $\mathcal{P}_{n_{b}, n_{s}}=\left((\mathcal{P})^{n_{b}} / S^{n_{b}}\right) \otimes\left((\mathcal{P})^{n_{s}} / S^{n_{s}}\right)$ quotient space defines only the number of buyers and sellers sitting at each point in the price space $\mathcal{P}$ (the "occupation numbers" $n_{x}^{b}$ and $n_{x}^{s}$ at $\left.x \in \mathcal{P}\right)$, and is thus defined by two summable sequences of positive integers on $\mathcal{P},\left\{n_{b}(x), x \in \mathcal{P}\right\} \in \mathcal{L}_{1}\left(\mathcal{P}, \mathcal{Z}^{+}\right)$(for the buyers) and $\left\{n_{s}(x), x \in \mathcal{P}\right\} \in \mathcal{L}_{1}\left(\mathcal{P}, \mathcal{Z}^{+}\right)$(for the

${ }^{\ddagger}$ This factor is not a constant on $\mathcal{P}^{n}$, and therefore this multinomial factor is in fact an operator, which we describe in the following subsections. 
sellers). Here $\mathcal{L}_{1}\left(\mathcal{P}, \mathcal{Z}^{+}\right)$is the space of positive-integer valued, summable sequences on $\mathcal{P}$. In other words, there is a bijection between $\mathcal{L}_{1}\left(\mathcal{P}, \mathcal{Z}^{+}\right) \otimes \mathcal{L}_{1}\left(\mathcal{P}, \mathcal{Z}^{+}\right)$and our full space of configurations $\mathcal{P}_{\text {Full }}=\cup_{n_{b}, n_{s}} \mathcal{P}_{n_{b}, n_{s}}$. This in turn defines a norm-preserving bijection between $\mathcal{M}\left(\mathcal{L}_{1}\left(\mathcal{P}, \mathcal{Z}^{+}\right) \otimes \mathcal{L}_{1}\left(\mathcal{P}, \mathcal{Z}^{+}\right)\right)$and $\mathcal{M}\left(\mathcal{P}_{\text {Full }}\right)$. This aspect of statistical field theory is much like the Garman model[[3]. Thus our states are elements of the measure space $\mathcal{M}\left(\mathcal{P}_{\text {Full }}\right)=\mathcal{M}\left(\mathcal{L}_{1}\left(\mathcal{P}, \mathcal{Z}^{+}\right) \otimes \mathcal{L}_{1}\left(\mathcal{P}, \mathcal{Z}^{+}\right)\right)$.

The Pure State Basis for the Space of State Measures, and Insertion and Deletion Operators

The foregoing describes the mathematical system required by our proposal to study liquidity scaling laws within the simplest possible market model. It turns out that the configuration space we have deduced above is identical to that of statistical field theory, used in physics and chemistry to describe systems with a variable number of particles. Below we include a brief qualitative introduction to statistical field theory. We have modified it slightly, following the work of Doi 31] and Peliti 32], in order to remove some specifically quantum mechanical features which appear in standard treatments of the field 47.

Statistical field theory is most easily introduced in a particular basis for the measure space, that of the "pure states". A pure state is an element of $\mathcal{M}\left(\mathcal{P}_{\text {Full }}\right)=\mathcal{M}\left(\mathcal{L}_{1}\left(\mathcal{P}, \mathcal{Z}^{+}\right) \otimes\right.$ $\mathcal{L}_{1}\left(\mathcal{P}, \mathcal{Z}^{+}\right)$) which has all of its probability mass concentrated at a single point of $\mathcal{P}_{\text {Full }}=$ $\mathcal{L}_{1}\left(\mathcal{P}, \mathcal{Z}^{+}\right) \otimes \mathcal{L}_{1}\left(\mathcal{P}, \mathcal{Z}^{+}\right)$. We may describe such a point with the sequences of occupation numbers $\left\{n_{x}^{b}\right\},\left\{n_{x}^{s}\right\}$ - denote this state as $\mid\left\{n_{x}^{b}, n_{x}^{s}: x \in \mathcal{P}\right\}>$. These states form a natural basis for $\mathcal{M}\left(\mathcal{L}_{1}\left(\mathcal{P}, \mathcal{Z}^{+}\right) \otimes \mathcal{L}_{1}\left(\mathcal{P}, \mathcal{Z}^{+}\right)\right)$. The usual $\mathcal{L}^{1}$ norm of a measure $\mu=$ $\left.\sum_{\left\{n_{x}^{b}, n_{x}^{s}\right\}} P_{\left\{n_{x}^{b}, n_{x}^{s}\right\}}\right\}\left\{n_{x}^{b}, n_{x}^{s}\right\}>$ in this space is simply $\|\mu\|=\sum_{\left\{n_{x}^{b}, n_{x}^{s}\right\}} P_{\left\{n_{x}^{b}, n_{x}^{s}\right\}}$.

Formally, this basis is constructed as follows, in the example of a discrete price space such as ours. Assume, to start with, that there is only one type of trader, say buyers, present in the market. We define the unique state $\mid 0>$ which is empty of traders, and linear operators $\Psi_{x}^{(B)^{\dagger}}, x \in \mathcal{P}$ which insert a buyer at $x, \Psi_{y}^{(B)^{\dagger}}\left|\left\{n_{x}: x \in \mathcal{P}\right\}>=\right|\left\{n_{x}: x \in\right.$ $\mathcal{P}, x \neq y$, and $\left.n_{x}+1, x=y\right\}>$. Then an element of the basis alluded to above is

$$
\left|\left\{n_{x}: x \in \mathcal{P}\right\}>=\prod_{x \in \mathcal{P}}\left(\Psi_{x}^{(B)^{\dagger}}\right)^{n_{x}}\right| 0>.
$$

These operators are known as buyer insertion, or buyer creation operators. In a similar way we introduce the buyer deletion, or buyer destruction operators $\Psi_{x}^{(B)}, x \in \mathcal{P}$, $\Psi_{y}^{(B)}\left|\left\{n_{x}: x \in \mathcal{P}\right\}>=\right|\left\{n_{x}: x \in \mathcal{P}, x \neq y\right.$, and $\left.n_{y}-1, x=y\right\}>$ if $n_{y}>0$, and 0 otherwise. The linear operators $\Psi_{x}^{(B)}$ and $\Psi_{y}^{(B)^{\dagger}}$ commute with each other unless $x=y$, in which 
case we have $\Psi_{x}^{(B)}{ }^{\dagger} \Psi_{x}^{(B)}-\Psi_{x}^{(B)} \Psi_{x}^{(B)^{\dagger}}=1$, in other words $\Psi_{x}^{(B)^{\dagger}} \Psi_{y}^{(B)}-\Psi_{y}^{(B)} \Psi_{x}^{(B)^{\dagger}}=\delta_{x y}$, where $\delta_{x y}$ is the Kronecker delta. (In the case where $\mathcal{P}$ is a continuous space, we write the above as $\Psi_{x}^{(B)^{\dagger}} \Psi_{y}^{(B)}-\Psi_{y}^{(B)} \Psi_{x}^{(B)^{\dagger}}=\delta(x-y)$, where the right hand side is Dirac's delta function.) For each $x \in \mathcal{P}$ we may define operators $N_{x}^{(B)}$ whose eigenvectors are the basis elements $\mid\left\{n_{x}: x \in \mathcal{P}\right\}>$, and whose eigenvalues are the occupation numbers $n_{x}^{b}$ as $N_{x}^{(B)}=\Psi_{x}^{(B)^{\dagger}} \Psi_{x}^{(B)}$. This operator goes by the name of the occupation number operator, but in our context it is sensible to call it the buyer number operator.

\section{The Norm on our Measure Space - a Single Kind of Trader}

With this in hand, we may define the appropriate statistical field theory norm $<\cdot>$ (due to Doi 31] and Peliti [32]) in terms of the function $\mathcal{F}$ on pure states $\mathcal{F}(\mu)=1$ if $\mu=\mid 0>$ and 0 otherwise, which we extend as a linear operator to all states. In terms of this function $\mathcal{F}(\cdot)$, we define $\left\langle\mu>=\mathcal{F}\left(\exp \left(\sum_{x} \Psi_{x}^{(B)}\right) \mu\right)\right.$. Here the exponential factor is precisely the operator that introduces the correct multinomial factors into the subspace measures, to compensate for overcounting of configurations in our representation of the configuration space as positive integer sequences on $\mathcal{P}$. Because of the presence of this factor, the averaged density of traders may be calculated as $\left\langle\Psi^{(B)^{\dagger}}(x) \Psi^{(B)}(x)\right\rangle=<$ $\Psi^{(B)}(x)>$. Note that the coefficients $P_{\left\{n_{x}^{b}\right\}}$ of the pure states are proportional to, but not equal to, the probability of the occurrence of that state. We refer to this as the "probability amplitude".

This treatment of Doi's work is different from that in his paper, so here we relate our exposition of Doi's work to his own. Statistical field theory is normally used in the context of quantum theory, which demands a conjugation operation, an $\mathcal{L}^{2}$ norm, and inner product, making the space a Hilbert space $\mathcal{H}: a, b \in \mathcal{H} \longrightarrow<a \mid b>\in \mathcal{C}$. Doi embeds his formulation inside this Hilbert space. He does this by introducing a special state $<S U M \mid$

$$
<S U M|=<0| \prod_{i} \exp \left(\sum_{x} \Psi(x)\right)
$$

with the property

$$
<S U M\left|\Psi^{\dagger}=<S U M\right|
$$

and he defines the $\mathcal{L}^{1}$ norm in terms of the $\mathcal{L}^{2}$ space inner product as $\|\Psi\|_{1}=<S U M \mid \Psi>$

The Norm on a Space with a Second Type of Trader: Sellers

We may of course have more than one type of trader in our system, and it is essential that we do. We introduce a basis containing a complete set of occupation states for sellers, 
so that a full basis for our measure space is $\mid\left\{n_{x}^{b}, n_{x}^{s}: x \in \mathcal{P}\right\}>$, and along with the buyer insertion and deletion operators $\Psi_{x}^{(B)^{\dagger}}, \Psi_{x}^{(B)}$, we introduce the seller insertion and deletion operators $\Psi_{x}^{(S)^{\dagger}}, \Psi_{x}^{(S)}$. Similarly we define the seller number operator $N_{x}^{(S)}=\Psi_{x}^{(S)^{\dagger}} \Psi_{x}^{(S)}$. The standard norm of a measure $\mu=\sum_{\left\{n_{x}^{b}\right\},\left\{n_{x}^{s}\right\}} P_{\left\{n_{x}^{b}\right\},\left\{n_{x}^{s}\right\}} \mid\left\{n_{x}^{b}\right\},\left\{n_{x}^{s}\right\}>$ in this expanded space is simply $\sum_{\left\{n_{x}^{b}\right\},\left\{n_{x}^{s}\right\}} P_{\left\{n_{x}^{b}\right\},\left\{n_{x}^{s}\right\}}$, and so the norm we will use for expectations is < $\mu>=\mathcal{F}\left(\exp \left(\sum_{x} \Psi_{x}^{(B)}\right) \exp \left(\sum_{x} \Psi_{x}^{(S)}\right) \mu\right)$

\section{We May Allow Trader Types with Several Different Kinds of Behavior}

In analogy with the case of sellers, we may introduce completely new species of traders, such as the market order traders of the two-fluid model, or traders with different dynamical behavior (e.g. momentum traders, as in the bias model, see section 4). We may even introduce other species still, which are not traders at all, but serve to communicate information between traders (see appendix). A species may have a completely different kind of dynamics, or even no dynamics at all. One important example of additional species that we shall need to introduce is the non-diffusing, externally controlled trader (either buyer or seller) which annihilates traders of the other type, but does not diffuse, and may not leave the market after trading (e.g. a specialist who sets a bid price and an offered price, good for any size, and stays in the market (see section 3$)$. . We denote these as $\Psi_{x}^{(\cdot),(e)}, \Psi_{x}^{(\cdot),(e)^{\dagger}}$, where $(\cdot)$ stands for either $(B)$ or $(S)$.

\section{The Fundamental Variables of the Theory are Operators on the State Measure}

The linear operators $\left.\Psi_{x}^{(\cdot)}, \Psi_{x}^{(\cdot)}\right)^{\dagger}$, and $N_{x}^{(\cdot)}$ are actually maps from the price space $\mathcal{P}$ into the set of linear operators on the measure space. Following the terminology of statistical field theory, we shall refer to such operators as "field operators" or "fields".

The $\Psi$ s and $\Psi^{\dagger}$ s are the building blocks of all computation in statistical field theory. Consider any market observable, e.g. the best bid $B$. It is possible to define (in terms of $\Psi$ s and $\Psi^{\dagger} \mathrm{s}$ ) an associated linear operator $\hat{B}$, that acts on pure states $\mid n_{x}>$ by multiplication by the best bid of that pure state. The action of this operator on the measure defining the state of our system $\mid$ state $>=\sum_{\left\{n_{x}\right\}} P_{\left\{n_{x}\right\}} \mid\left\{n_{x}\right\}>$ gives another measure $|b>=\hat{B}|$ state $>$, whose expansion coefficients in the pure state basis is the best bid of the pure state times its probability amplitude in $\mid$ state $>:\left|b>=B_{\left\{n_{x}\right\}} P_{\left\{n_{x}\right\}}\right|\left\{n_{x}\right\}>$. Its norm is therefore equal to the expectation value of the best bid $<B>_{t}=\| \hat{B} \mid$ state, $\mathrm{t}>\|$

We may use this procedure for evaluating any desired expectation value in statistical field theory. We construct the appropriate operator using the $\Psi_{\mathrm{s}}$ and $\Psi^{\dagger} \mathrm{s}$, apply this operator to our (time dependent) state measure $\mid$ state, $t>$, and evaluate its norm, thus 
$<$ Op $>=\|$ Ôp $\mid$ state, $t>\|$. We construct several of these operators in the next section.

We shall use this procedure throughout this paper, but in a modified form. The time evolution operator $U_{t, t^{\prime}}$ satisfies $\mid$ state, $t^{\prime}>=U_{t, t^{\prime}} \mid$ state, $t>$. Thus, the expectation of any observable $A$ at a time $t$ is $<A>_{t}=\| \hat{A} \mid$ state, $t>\|=\| \hat{A} U_{t, 0} \mid$ state, $0>\|=$ ||$U_{t, 0}^{-1} \hat{A} U_{t, 0} \mid$ state, $0>\|$. We define the time dependent version of an operator $A$ via its conjugation with $U: \hat{A}(t)=U_{t, 0}^{-1} \hat{A} U_{t, 0}$. We may write operator expectations as $\langle A\rangle_{t}=$ $\| \hat{A}(t) \mid$ state, $0>\|$.

\section{Dimensional Analysis and the Parameters of Diffusion-Controlled Annihilation}

Diffusion-annihilation dynamics consists of diffusions interspersed with annihilations, for each species of trader. We may construct both a diffusion operator $\mathcal{D}_{(\cdot)}$ and an annihilation operator $\mathcal{U}_{\text {ann }}$ from the set $\left\{\Psi_{x}^{(\cdot)}, \Psi_{x}^{(\cdot)}{ }^{\dagger}\right\}, x \in \mathcal{P}$, in order to assemble a diffusionannihilation operator that evolves a state forward in time within our model. Although we shall postpone a discussion of these operators to the appendix, we shall make one important comment about the dynamics of the model. The operator evolution equation ( see appendix 7.1) may be differentiated to yield an operator differential equation. In general, if a partial differential equation is invariant under the change of dependent variables $x \longrightarrow \alpha x, t \longrightarrow \beta t$, then its solutions are also invariant, and this is a powerful constraint on the form of its solutions. More commonly, however, PDEs are changed into a different, but similar equations. However, this invariance property may be given to any PDE at all, provided that there are constants in front of each term that are defined to scale in the appropriate way under the abovementioned transformation, and by this method we may infer strong constraints on the way the solution depends on these constants, those in the boundary conditions, and the dependent variables $t$ and $x$. The scaling of these constants is known as their dimensions, and the (generally very simple) analysis to determine the constraints they imply on the solutions is known as dimensional analysis.

In our case, there is only one coefficient necessary in the PDE, the diffusion coefficient $D$, with dimensions $x^{2} / t$, i.e. $D \sim(\text { dollars })^{2} /(\mathrm{sec})$. In addition, there is a dimensionful constant in the boundary conditions, $J$, the rate at which traders enter the market at the boundaries, which in a steady state market is equal to the deal rate, with dimension $J \sim 1 /(\mathrm{sec})$. Thus the expectation $\langle X\rangle$ of any quantity $X$ with dimensions $[X]=$ $(\text { dollar })^{m} / \sec ^{n}$ must be proportional to $(D / J)^{m / 2} J^{n} \sim J^{n-m / 2}$. In particular, lengths in the model scale as $\sqrt{D / J}$. As the foregoing analysis has been for a continuous price space, note that the quantities $\langle X\rangle$ must converge to something well-defined in the continuum limit. 
In the foregoing, we have defined the number operator $N^{(\cdot)}(x)$, an operator which gives the number of traders sitting at point $x$, in a pure state. It is, of course, dimensionless. This operator is very closely related to another operator, the density operator $\rho^{(\cdot)}(x)$, which gives the number of traders per unit length near point $x$. They are related as $\rho^{(\cdot)}(x)=N^{(\cdot)}(x) / \delta S$, where $\delta S$ is the lattice spacing. In this case it is the trader density $<\rho(x)>$, and not $\langle N(x)>$, which has a good continuum limit, and so $<\rho>\sim \sqrt{J / D}$

Note that our discussion of dimensional analysis has ignored the lattice spacing $\delta S$, which is actually another dimensionful quantity on which these scalings may depend. The length of the price space $L$ has also been omitted. These two represent the lower and upper limits of the length scales in our model, and if we were to imagine a Fourier analysis of the dynamics, most of the dynamics takes place far from these two scales, in the intermediate regime. As a result, dynamical quantities tend to depend slowly at best on these quantities, often adding logarithmic corrections to scaling laws. As a result, it is correct, generally speaking, to ignore these length scales in a dimensional analysis of scaling behavior. Numerical simulations of this model [30] have borne out these arguments.

\section{Parametrizations of the Reaction Front}

A model of this kind allows us to calculate the relationships described in the introduction, but also many more. In general, if we start this model with any initial state satisfying the initial condition above, it will evolve to a state with a "reaction front". This is a region within the state where the buyers meet the sellers. At the center of the reaction front is, of course, the best bid, best offer and midmarket. Beyond the best bid/best offer we expect to see an increasing density of buyers/sellers.

A stationary reaction front corresponds to an approximately steady state situation similar to the ground state introduced in the previous section. Buyers and sellers approach each other, meet near the center, and annihilate. At the same time, traders are injected into the system at a rate equal to the deal rate.

The statistics that we read off of a trading screen describe the shape and dynamics of the reaction front - the best bid locates the top of the lower edge and the best offer the bottom of the upper edge (see Figures 1 and 3). The bid size tells us the height at the best bid point, and the offer size tells us a similar thing about the best offer point. And the last trade field tells us about its recent history.

Generally we are interested in all parameters which describe this reaction front, its 
shape and its dynamics. The bid-offer spread is but one, albeit the most important, of the parameters describing the shape of the reaction front.

Within a pure state, we may describe the shape fully, at a time $t$, by specifying the number of buyers $\rho_{B}(x, t)$ and sellers $\rho_{S}(x, t)$ at each point $x$ of the price space. Obviously $\rho_{B}(x, t)=0$ for $x$ greater than the best bid, and $\rho_{S}(x, t)=0$ for $x$ less that the best offer. The number of sellers between $x_{\ell}$ and $x_{u}$ is then $\sum_{x=x_{\ell}}^{x_{u}} \rho_{S}(x, t)$.

Equivalently, we may describe the shape by specifying, for all $n$, the point $X_{B}(n, t)$ in price space above which exactly $n$ buyers may be found, and the point $X_{S}(n, t)$ below which exactly $n$ sellers may be found. $X(n, t)$ is the inverse of the function $f(x, t)=$ $\sum_{x^{\prime}=0}^{x} \rho\left(x^{\prime}, t\right)$. The best bid $B(t)$ is then $B(t)=X_{B}(1, t)$, and the best offer $O(t)$ is then $O(t)=X_{S}(1, t)$. And we may interpret $X_{B}(n, t)$ as the tender price necessary to make $n$ purchases (immediately), and the price paid for the $n$ purchases would be $\sum_{x}^{X_{S}(n, t)} x \rho_{S}(x, t)$.

We may then express the density of traders near the best bid as $S_{B}$ as $S_{B}(t)=$ $\rho_{B}(B(t), t)$, and the density near the best offer $S_{O}$ similarly as $S_{O}(t)=\rho_{S}(O(t), t)$. Of course, the bid-offer spread is then $\operatorname{Spr}(t)=O(t)-B(t)$, and the midmarket is $M(t)=$ $(1 / 2)(B(t)+O(t))$. Note that $B(t), S(t)$, and $M(t)$ are not Markov random variables, and do not satisfy a stochastic differential equation, because they are subject to jumps.

Finally, the trading screen describes more than just the shape of the reaction front, because it also describes the history of trades, through the last trade price $\chi(t)$, and through the instantaneous deal rate (trading volume/sec) $J$. We show how these are parametrized in the appendix.

\section{Digression on the history of Diffusion-Controlled Annihilation in the phys- ical and chemical literature.}

The foregoing arguments have established the nature of our model, commonly known in the physics and chemistry literature as steady state diffusion- driven annihilation in one dimension. We pause here to mention previous work on this and related subjects. As previously mentioned, our model considers a steady state market, in which buyers and sellers are injected into the system at a rate $J$ which precisely compensates the losses due to annihilations. However, one can also consider the nonstationary situation in which no traders are injected to compensate for the annihilations - in this case the width of the reaction front grows with time. In the initial paper by Gálfi and Rácz [28] the properties of the reaction front in a system with segregated initial conditions were studied in the 
mean field approximation. The mean field approximation is one which approximates the state by a Gaussian centered on the most heavily weighted configuration, and ignores higher, non-Gaussian corrections, known as "fluctuations". It works well for dynamics in three dimensional space, but breaks down in lower dimensions due to the important role of microscopic density fluctuations in one and two-dimensional systems and in one dimension their influence is particularly important 3. Numerical simulations were performed in [30], and showed, among other things that reaction rates are a Gaussian function of $x \in \mathcal{P}$. Analytical calculations by Cardy et. al. [29] confirmed these numerical results.

Let us also note the paper by Bak, Paczuski and Shubik (BPS) 27] where a model based on diffusion-controlled annihilation was first applied to the stock market. These authors introduce a series of models based on diffusion-controlled annihilation as a route towards recovering the observed Levy-Pareto "fat-tail" distributions which are said to describe the medium term evolution of the stock-market. The diffusion-controlled annihilation process is modified by various forms of sociological interaction, similar in philosophy to our bias model. It is further modified by the presence of so-called "rational traders" each with a different strategy, that set their bids and offers according to their own individual expectations. The rational traders in this model are non-identical, whereas the nonrational "noise" traders are identical to one another, and so the pure noise trader case, which they discuss at length, has the same dynamics as our minimal model. Buyers who are deleted from the market, however, are re-inserted randomly as sellers, and vice versa. The full BPS model, from which they obtain their best results, gets its unique and interesting dynamics from the various different (history dependent) strategies which must wrestle with one another in order to find a (possibly non-existent) equilibrium. This model, while based on diffusion-controlled annihilation, is quite different from our model, and certainly more complicated. Nevertheless, the success of a model based on diffusioncontrolled annihilation in reproducing observed statistics of trade prices provides some encouragement for our rather different, but related project of liquidity scaling laws.

\section{The Scaling Laws.}

We now proceed to a description of each of the research problems to which we alluded

$\S$ In a series of papers Cardy et. al. [29] shows how this obstacle may be partially overcome, and we shall use his method in the Analytical Results section 
in the introduction. We write down an expression which computes each scaling law in terms of the expectations of the operators in the previous section. Where necessary, we provide background, or define additional terms and notation. We write down what qualitative intuition tells us about the limits of the scaling law. In addition, for a few of the simpler problems, dimensional analysis is sufficient to compute the scaling laws directly, or a scaling form (for the minimal model) and we include these formulas as well. In these cases, the consistency of intuition with the results of dimensional analysis provides a satisfying and nontrivial check on our method. More generally, of course, these scaling laws will be evaluated by numerical simulation and we will report on these results in a future publication 45].

In the introduction it was mentioned that there are several other problems beyond the liquidity scaling laws which we may investigate with this model, leading to scaling laws beyond those discussed in the first section. We would like to draw the reader's attention to this point. This is, in fact, one of the most appealing features of this arena of research - there is a very large and diverse set of financially meaningful scalings that are easy to measure, easy to calculate, and should only require a simple statistical model, such as the models discussed in this paper, to make reasonably accurate predictions. As with the liquidity scaling laws, there is an obvious qualitative intuition which we may use as a guide and sanity check.

Furthermore, these additional scalings are not empty mathematical abstractions. Their qualitative behavior is readily apparent to market practitioners, and they are readily measured - they are correlations of quantities such as the best bid, best offer, trader density 冈, trade price, etc. which are directly observable in the marketplace.

\section{Liquidity Scaling Laws}

Liquidity in markets is measured in several different ways, using several different quantities, as proxies. The most common are the deal rate $J$, the time to midmarket sale, the bid-offer spread, and the trader densities near the best bid/offer. As these quantities all measure the same thing, they must all be monotonic functions of one another. We list these relations here, expressed as expectations of field operators discussed in the previous section, evaluated in the measure which is the steady state solution of our dynamics. This state measure depends on the deal rate $J$, and we include whatever information might be inferred about this dependence from dimensional analysis.

*this quantity can not usually be read off of a trade screen reliably, but may be observed by active intervention into the market. 
- Bid Offer Spread

$$
\operatorname{Spr}=<O(t)-B(t)>
$$

- Trade price variance

$$
w^{2}=<\chi^{2}(t)>-<\chi(t)>^{2}
$$

- Fluctuations in the Bid-Offer Spread

$$
(\Delta \mathrm{Spr})^{2}=<(O(t)-B(t))^{2}>-<O(t)-B(t)>^{2}
$$

We expect both $\operatorname{Spr}(J)$ and $w^{2} \longrightarrow 0$ as $J \longrightarrow \infty$. Both the bid offer spread and $w$ have dimension of dollars, so dimensional analysis suggests $\operatorname{Spr}(J) \sim \sqrt{D / J}, w(J) \sim$ $\sqrt{D / J}$ consistent with intuition. Using the results of 29] we shall show in the next section that there is a logarithmic correction to the the results of dimensional analysis, so that we have $w(J) \sim J^{-1 / 2} \ln J$ for finite size price space. Cardy's method will further enable us to calculate the auto-correlation function of trade prices $W(\tau)=<\chi(t+\tau) \chi(t)>-<\chi(t)><\chi(t+\tau)>$. In addition, it is possible, and possibly quite informative, to measure the fluctuations in the spread $\Delta$ Spr. This has the same dimensions as the spread itself, and so the same scaling law.

- Time to MidMarket Sale/Purchase (with certainty $1-\epsilon$ )

$$
\tau_{S}=g(1-\epsilon) ; \quad f(\tau)=<J_{\text {cum }}^{e}(t+\tau) \Psi_{e}^{\dagger}(M(t), t)>; \quad g(f(x))=x
$$

$\Psi_{e}^{\dagger}(M(t), t)$ inserts a non-diffusing trader at the mid market, who trades without ever leaving the market, i.e. he annihilates his counterparty, but not himself. $J_{\text {cum }}^{(e)}(t)$ is the cumulative deal rate operator, which computes the number of deals transacted with the trader $(e)$ during time interval $t$ (see Appendix). This quantity evaluates the average time he must wait before he is hit or lifted. This is not the only way to compute $\tau_{S}$, however. Another way to calculate $\tau_{S}$, for example, is to study the decay rate of an excited state of a steady state market with a $\Psi_{e}^{\dagger}(M(t), t)$ inserted at time $t$ :

$$
d / d t \ln <\Psi_{e}^{\dagger}(M(t), t)>=-\left(1 / \tau_{S}\right)
$$


We expect $\tau_{S} \longrightarrow 0$ as $J \longrightarrow \infty$. Because the dimension of $\tau_{S}$ is sec, dimensional analysis suggests $\tau_{S} \sim J^{-1}$, consistent with intuition.

A similar quantity was considered in the framework of a continuous price space by Cohen-Maier-Schwartz-Whitcomb [8], who showed that the probability of a limit order trade does not go continuously to 1 as a putative limit order bid approaches the best offer. This came about because the random process for the best offer was taken to be a finite sum of $N$ Poisson processes, approaching a Wiener process in the limit $N \longrightarrow \infty$. Under these assumptions, the anomalous probability behavior, and the existence of a non-infinitesimal bid-offer spread was proved, which however vanishes in the Wiener limit. Because our traders are considered to readily change their bid and offer prices, the analysis of Cohen-Maier-Schwartz-Whitcomb would require $N$ very large, near to the Wiener limit, leading us to expect the probability of trading to go to 1 as a limit order bid is placed near to the best offer. In both models, $\tau_{S} \longrightarrow 0$ as $J \longrightarrow \infty$.

- Density near the best bid/offer as a function of deal rate

For a small intervals or price space $\Delta p, \Xi_{B}(x, t)$ represents the number bids we can hit in the vicinity of the price, between $B(t)+x$ and $B(t)+x+\Delta p$

$$
\begin{array}{cc}
\hat{\Xi}_{B}(x, t)=\rho_{B}(B(t)+x, t) & \hat{\Xi}_{O}(x, t)=\rho_{O}(O(t)+x, t) \\
\Xi_{B}(x, t)=<\hat{\Xi}_{B}(x, t)> & \Xi_{O}(x, t)=<\hat{\Xi}_{O}(x, t)>
\end{array}
$$

This quantity provides to us information about the ease with which we can move the market, and the transaction costs of trading in large volume all at once. We expect $\Xi_{B}, \Xi_{O} \longrightarrow \infty$ as $J \longrightarrow \infty$. Dimensional analysis leads to the scaling law $\Xi_{(\cdot)}(0) \sim$ $(J / D)^{1 / 2}$, and $\Xi_{(\cdot)}(\Delta x)$ has the scaling form $\Xi_{(\cdot)}(\Delta x)=(J / D)^{1 / 2} f\left(J \Delta x^{2} / D\right)$.

The expressions above are the mathematical formulation of the qualitative laws discussed in the introduction. In a future publication, we shall go beyond simple dimensional analysis, and report on results from actual numerical simulations of the model 45.

\section{Higher Correlation Functions Describing Equilibrium}

Beyond scaling laws directly associated with liquidity, there are many others which explore the dynamical behavior of an equilibrium market. These quantities are normally 
associated with correlations of operators at different times, and/or conditioning of the expectation. The time differences $\Delta t_{i}$ between the different operators, and the conditioning parameters now enter our calculations as new dimension-ful parameters. This weakens the power of dimensional analysis, because there is no longer a single, unique dimensionful combination of parameters which matches the dimensions of the operator we are averaging. Thus, for example, correlation functions will have different $J$ dependence for small $\tau<<1 / J$ and large $\tau>>1 / J$. One may consider following functions:

- Correlation of Changes in Best Bid with Changes in Best Offer

For any quantity $f(t)$, define $\Delta_{\tau} f(t)=f(t+\tau)-f(t)$.

$$
C_{\Delta_{t} B \Delta_{t} O}(\tau)=\frac{<(B(t+\tau)-B(t))(O(t+\tau)-O(t))>}{\sqrt{<(B(t+\tau)-B(t))^{2}><(O(t+\tau)-O(t))^{2}>}}
$$

When the best bid snaps back after a trade, it moves down. When, at the same moment the best offer snaps back it moves up. Afterwards, the best bid resumes diffusing upward, while the best offer then diffuses back downward. We therefore expect that, if we look forward only over time scales much shorter than one dealing time, $\tau_{D} \sim 1 / J$

$$
C_{B O}(\tau) \longrightarrow \frac{<d B(t) / d t d O(t) / d t)>}{\sqrt{<d B(t) / d t>^{2}<d O(t) / d t>^{2}}} \longrightarrow-1
$$

Dimensional analysis suggests that $<d B(t) / d t d O(t) / d t)>\sim-D J$

But when we look forward over many dealing times, the market will have moved to one centered around a completely new midmarket, and the best bid and best offer will follow, together. For these time scales $\tau>>1 / J, C_{B O}(\tau) \longrightarrow 1$, if that new midmarket is centered sufficiently far away.

- Density at/near Best Bid/Offer conditioned on Fluctuations in Best Bid/Offer:

$$
\begin{gathered}
\Xi_{B}\left(\sigma_{B}\right)=\frac{<\rho_{B}(B(t), t) I\left((B(t)-\bar{B})^{2}=\sigma_{B}^{2}\right)>}{<I\left((B(t)-\bar{B})^{2}=\sigma_{B}^{2}\right)>} \\
=E\left(\rho_{B}(B(t), t) \mid(B(t)-\bar{B})^{2}=\sigma_{B}^{2}\right)
\end{gathered}
$$

$I(\cdot)$ is the indicator function. (For simplicity, we discuss only the bid - the offer side case is, of course, identical). If the density of buyers is higher, then we can expect 
that the best bid does not need to snap back as far when it jumps back to the next best bid. We therefore expect that $\Xi_{B}$ will be small at large $\sigma_{B}$, and large at small $\sigma_{B}$. As $\sigma_{B}$ represents an additional dimension-ful parameter, with dimensions of (dollars), we cannot use dimensional analysis to infer the $J$ dependence of $\Xi_{B}\left(\sigma_{B}\right)$, however we do know that it will be of the form $\Xi_{B}\left(\sigma_{B}\right)=\sqrt{J / D} f\left(\frac{D}{J \sigma_{B}^{2}}\right)$. Here $f$ is an unknown function of a single variable, whose expansion in its argument must contain only negative powers. We may generalize this quantity to explore the density beyond the best bid/offer, as follows.

$$
\Xi_{B}\left(\sigma_{B}, \Delta x\right)=\frac{<\rho_{B}(B(t)+\Delta x, t) I\left((B(t)-\bar{B})^{2}=\sigma_{B}^{2}\right)>}{<I\left((B(t)-\bar{B})^{2}=\sigma_{B}^{2}\right)>}
$$

This is a more complicated quantity, whose Laurent expansion coefficients may be treated in a similar way.

- Time Change in Spread conditioned on Spread: We define the conditional expectation

$$
\Delta_{\tau} \operatorname{Spr}\left(s_{0}\right)=\frac{<\left(\Delta_{\tau} \operatorname{Spr}(t) I\left(\operatorname{Spr}(t)=s_{0}\right)>\right.}{<I\left(\operatorname{Spr}(t)=s_{0}\right)>}=E\left(\Delta_{\tau} \operatorname{Spr}(t) \mid \operatorname{Spr}(t)=s_{0}\right)
$$

where $I(\cdot)$ is the indicator function. The spread in a double auction market starts out wide, then narrows slowly until the bid and offer meet, at which point a deal is made and the best bid and offer snap back. Thus the expected change in time of the spread $(\Delta \operatorname{Spr}(\mathrm{t}) \operatorname{Spr}(t+\tau)-\operatorname{Spr}(t))$ conditioned on the value of the spread is small and negative for large values of the spread, and large and positive for small values of the spread. The zero point of this function may be interpreted as the average width at which a deal becomes imminent (the price is "irresistible"). When $\tau$ is close to zero, so that the expectation is a derivative, there is a scaling form for this quantity as well $-\Delta_{t} \operatorname{Spr}\left(s_{0}\right) \sim \sqrt{D / J} f\left(\frac{D}{J s_{0}^{2}}\right)$, where again $f$ is an unknown function of a single variable. Because of our intuition that $\Delta_{t} \mathrm{Spr}$ increases sharply as the value $s_{0}$ of Spr decreases, it is reasonable to expect that the unknown function $f(x) \longrightarrow \infty$ as $x \longrightarrow \infty$.

\section{Market Response to a Disequilibrium of Buyers and Sellers}

When shocked into a state of disequilibrium, a market will go through changes to its internal structure as it finds a new equilibrium. We may study the characteristic rates and magnitudes of these changes, to each of the several parameters describing an equilibrium market. 
- Response Functions to Density Imbalance: We define the conditional expectations

$$
\begin{gathered}
R_{B}(t, \tau)=\frac{<\Delta_{\tau} B(t) I\left(\rho_{O}(O(t), t)-\rho_{B}(B(t), t)=\Delta \rho\right)>}{<I\left(\rho_{O}(O(t), t)-\rho_{B}(B(t), t)=\Delta \rho\right)>} \\
=E\left(\Delta_{\tau} B(t) \mid \rho_{O}(O(t), t)-\rho_{B}(B(t), t)=\Delta \rho\right) \\
R_{O}(t, \tau)=\frac{<\Delta_{\tau} O(t) I(\rho(O(t), t)-\rho(B(t), t)=\Delta \rho)>}{<I(\rho(O(t), t)-\rho(B(t), t)=\Delta \rho)>} \\
R_{J}(t, \tau)=\frac{<\Delta J(t) I(\rho(O(t), t)-\rho(B(t), t)=\Delta \rho)>}{<I(\rho(O(t), t)-\rho(B(t), t)=\Delta \rho)>}
\end{gathered}
$$

where $I(\cdot)$ is the indicator function. From these response functions we may derive characteristic parameters for the market's response to a small disturbance, such as the response time $\tau_{R}$ to a density imbalance, or the expected size of the movement, of any one of the response functions, e.g. $R_{B}$, due to a density imbalance $\Delta R_{B}=$ $R_{B}(t, \tau)-\lim _{\tau \rightarrow 0} R_{B}(t, \tau)$. For $\tau_{R}$ we expect that, as $J \longrightarrow \infty, \tau \longrightarrow 0$, and dimensional analysis suggests that $\tau_{R} \sim J^{-1}$. For $\Delta R_{B}$, we expect that as $J \longrightarrow \infty, \Delta R_{B} \longrightarrow 0$, and as $\tau \longrightarrow \infty, \Delta R_{B} \longrightarrow$ finite. In addition, as $\tau \longrightarrow 0$, this quantity becomes a derivative times $\tau$, so that $\Delta R_{B} \sim \tau \sqrt{D J}$, and as $J \longrightarrow 0$, $\Delta R_{B} \longrightarrow \infty$. Dimensional analysis suggests the scaling form $\Delta R_{B} \sqrt{D / J} f(J \tau)$, with $\lim _{x \rightarrow \infty} f(x)=$ finite, and $\lim _{x \rightarrow 0} \sim x . \Delta R_{B} \sim J^{-1}$. Of particular interest is the final resting place of the best bid, $\Delta R_{B}(\tau=\infty)$.

\section{Transaction Costs}

- Expected Profits for a Specialist: Given a Bid Offer Spread set by a specialist, what is the expected profit or loss?

This problem has been studied in the context of market makers by [3, 4, 5, 6, 11, 21, 12]. Here we study the specialist problem, using our diffusion-annihilation model. A specialist sets a bid and an offer price in the market to provide liquidity, and makes a profit from the spread, just as a market maker does. However, he doesn not monopolize trading - other limit order traders may trade with each other. This

${ }^{\dagger}$ In fact, as it is reasonable to expect a response to density imbalance proportional to the magnitude, we may expect an exponential behavior in the response function, and may in that case define $\tau_{R}$ as $\tau_{R}^{-1}=-d / d \tau \ln R(t, \tau)$. 
problem therefore retains the difficulties of a double auction market and requires treatment by statistical field theory. A full treatment also requires consideration of the effects of inventory, asymmetric information, and other traditional aspects of the market maker problem.

A specialist sets the width and midmarket of his quoted prices, in order to maximize profits and minimize risk. Define the net profit/loss as the sum of all of netted out positions $P_{N}(t)$ after time $t$, and define a second quantity to be the net open position $P_{O}(t)$ after time $t$.

$$
\begin{gathered}
f_{i}=J_{\operatorname{cum}}^{\left(e_{i}\right)}\left(t^{\prime}\right) \Psi_{B\left(e_{1}\right)}^{\dagger}(M(t)-\Delta B, t) \Psi_{S\left(e_{2}\right)}^{\dagger}(M(t)+\Delta S, t) \\
P_{N}=\min _{i=1,2} f_{i} \quad P_{O}=\left(f_{2}-f_{1}\right)
\end{gathered}
$$

$\Psi^{\dagger}$ inserts an external non-diffusing trader, who trades without ever leaving the market, ie.e he annihilates his counterparty, but not himself. $J_{\text {cum }}^{\left(e_{i}\right)}$ measures the total volume of trades for the trader $\left(e_{i}\right)$.

We define a good market-making strategy as one that results in a positive expected profit, and zero expected net open position, i.e. $\left\langle P_{O}(t)>=0,\left\langle P_{N}(t)>>0\right.\right.$ for all $t$. In addition, we may define the fluctuations in both of these quantities $<P_{O}^{2}(t)>-<P_{O}(t)>^{2}$, and $<P_{N}^{2}(t)>-<P_{N}(t)>^{2}$. They define the two sources of uncertainty for a market making strategy, and we may use them to measure the risk/reward ratio of a particular strategy for setting the market maker's bid and offer.

- Tender Offer:

How far above the best offer $\Delta x_{N}$ must we bid in order to attract a given number of sellers $N$ within a specified time $t_{T}$, with certainty $1-\epsilon$ ?

The operator expectation we must construct is very similar to the one in the item above.

$$
\begin{gathered}
f(\Delta x)=<J_{\text {cum }}^{(e)}(t+\Delta t) \Psi_{B(e)}^{\dagger}(O(t)+\Delta x, t)+\sum_{O(t)}^{O(t)+\Delta x} \delta S \rho_{B}(O(t)+s)> \\
\Delta X_{N}(\Delta t)=f^{-1}(\epsilon)
\end{gathered}
$$


$\Psi_{B(e)}^{\dagger}$ inserts an external non-diffusing trader who trades once, and disappears from the market. $J_{\text {cum }}^{(e)}$ measures the total value of the trades done through this trader. Recall that $\delta S$ is the lattice spacing. For $\Delta t=0$, this quantity simplifies greatly, to just

$$
\Delta X_{N}(\Delta t=0)=<X_{B}(N, t)>
$$

which scales as $\sqrt{D / J}$.

We expect that, as $J \longrightarrow \infty, \Delta X_{N} \longrightarrow 0$, and conversely, as $J \longrightarrow 0, \Delta X_{N} \longrightarrow \infty$. We also expect that, when $\Delta t \longrightarrow \infty, \Delta X_{N} \longrightarrow$ finite, and $\Delta t \longrightarrow 0, \Delta X_{N} \longrightarrow<$ $X_{B}(N, t)>$, and that $\Delta x_{N}$ is monotonic.

Dimensional analysis predicts that $\Delta X_{N}(\Delta t)$ has the scaling form $\Delta X_{N} \sim \sqrt{D / J} g(J \Delta t)$, and this implies that $g(x)$ is finite and monotonic in both the limits $x \longrightarrow 0$, and $x \longrightarrow \infty$. Note that the consistency of these limits is a nontrivial check on the dimensional analysis result.

\section{Analytical Results}

Thus far we have discussed the predictions of our model in terms of computer simulations and dimensional analysis. In addition, however, many analytical approximation schemes also have been developed for statistical field theory. Although numerical results will be more precise, these methods supplement numerical simulations of the model by providing intuition.

Cardy et. al. 29 has developed one such approximation using a method known as mean field theory, in the context of a model of chemical reactions in one dimension. Mean field theory replaces the evolution equations for the operators with a partial differential equation for the density configuration with the greatest probability mass, ignoring others that may be contained within the state (known as the "fluctuations" away from the mean field). This scheme is particularly interesting because he retains some of he effects of the fluctuations by deriving an effective noise term. Mean field theory, however, is not a systematic approximation scheme - it has no system of higher order corrections, and there is no known error estimate. Furthermore, the scheme allows us only to calculate a small subset of the scaling laws of the previous section. However, its predictions coincide nicely with numerical simulations 29, 30.

A model of chemical reactions must differ from a model of market microstructure in certain respects. First, the configuration space for a chemical species can only be 
continuous, in contrast to our discrete price space $\mathcal{P}$. Also, chemical reactions differ from deals in a marketplace, in that they occur with only a finite probability per unit time $\lambda$ when the reactants have been joined, whereas exchange rules guarantee an immediate trade when a bid coincides with an offer. Thus the chemical model has the following time evolution operator

$$
\begin{gathered}
U_{\lambda}\left(t, t^{\prime}\right)=\exp H_{\lambda}\left(t^{\prime}-t\right) \\
H_{\lambda}=\int_{-L / 2}^{L / 2} d x\left[\bar{\Psi}_{b}\left(-D \nabla^{2} \Psi_{b}\right)+\bar{\Psi}_{s}\left(-D \nabla^{2} \Psi_{s}\right)+\right. \\
\left.\lambda\left(\bar{\Psi}_{b} \bar{\Psi}_{s}-1\right) \Psi_{b} \Psi_{s}-\bar{\Psi}_{b} J \delta(x+L)-\bar{\Psi}_{s} J \delta(x-L)\right]
\end{gathered}
$$

which may be derived from a master equation describing the evolution of probability distributions (see for example [29] and references therein, see also the Appendix). The first two terms describe diffusion of $B$ and $S$ particles, the next two terms describe annihilation $B+S \rightarrow 0$ and the last two terms are necessary to insert $B$ and $S$ particles at the edges of the system at the rate $J$. The size of the system is $L$, and our model corresponds to the limit $\lambda \longrightarrow \infty$.

In this section we shall outline Cardy et. al. method, and give several results for the minimal model. In later sections, where possible, we shall also present results for some of the extended models.

In general, mean field theory leads to a system of scalar partial differential equations. These are obtained as the extrema of a functional $S_{\lambda}$ of the fields $\bar{\Psi}_{b}(x, t), \bar{\Psi}_{s}(x, t), \Psi_{b}(x, t)$ and $\Psi_{s}(x, t), S_{\lambda}\left[\bar{\Psi}_{b}, \bar{\Psi}_{s}, \Psi_{b}, \Psi_{s}\right]$, which is associated with the evolution operator (3.18)

$$
\begin{array}{r}
S_{\lambda}=\int_{t^{\prime}}^{t} d t \int_{-L / 2}^{L / 2} d x\left[\bar{\Psi}_{b}\left(\frac{\partial \Psi_{b}}{\partial t}-D \nabla^{2} \Psi_{b}\right)+\bar{\Psi}_{s}\left(\frac{\partial \Psi_{s}}{\partial t}-D \nabla^{2} \Psi_{s}\right)+\right. \\
\left.\lambda\left(\bar{\Psi}_{b} \bar{\Psi}_{s}-1\right) \Psi_{b} \Psi_{s}-\bar{\Psi}_{b} J \delta(x+L)-\bar{\Psi}_{s} J \delta(x-L)\right]
\end{array}
$$

$\ddagger$ This operator differs from that given in the appendix in that it corresponds to

$$
\exp \left(H_{\text {Diff }}^{(B)}\left(t-t^{\prime}\right)+H_{\text {Diff }}^{(S)}\left(t-t^{\prime}\right)+H_{\text {ann }}\left(t-t^{\prime}\right)\right)
$$

instead of

$$
\exp \left(H_{\text {Diff }}^{(B)}\left(t-t^{\prime}\right)+H_{\text {Diff }}^{(S)}\left(t-t^{\prime}\right)\right) \exp H_{\text {ann }}\left(t-t^{\prime}\right)
$$

Because the arguments of the exponential are operators, these are not the same, and in particular, it not yet known whether this allows for some overlap of buyers and sellers, even in the limit $\lambda \longrightarrow \infty$, whereas it is explicit in the form given in the appendix. 
These partial differential evolution equations can be obtained by varying with respect to $\bar{\Psi}_{b, s}$ after a change of variables, the redefinition $\bar{\Psi}_{b, s} \rightarrow \bar{\Psi}_{b, s}+1$. Neglecting the nonlinear term $\lambda \bar{\Psi}_{b} \bar{\Psi}_{s} \Psi_{b} \Psi_{s}$ one gets the standard reaction rate equations for the densities $\rho_{B, S}(x, t)=<\Psi_{b, s}(x, t)>$

$$
\begin{array}{r}
\left(\partial_{t}-D \partial_{x}^{2}\right) \rho_{B}+\lambda \rho_{B} \rho_{S}-J \delta(x+L)=0 \\
\left(\partial_{t}-D \partial_{x}^{2}\right) \rho_{S}+\lambda \rho_{B} \rho_{S}-J \delta(x-L)=0
\end{array}
$$

and one finds a particularly simple equation for the quantity $\zeta(x, t)=\rho_{B}(x, t)-\rho_{S}(x, t)$, which may be interpreted as the density difference of buyers and sellers.

$$
\frac{d \zeta}{d t}-D \nabla^{2} \zeta=0
$$

This equation is independent of the reaction rate $\lambda$, so the limit $\lambda \longrightarrow \infty$ is obviated. It can be shown [29] that to take into account fluctuations one has to take into account the nonlinear term $\bar{\Psi}_{b} \bar{\Psi}_{s} \Psi_{b} \Psi_{s}$ which effectively adds a noise term in the right hand part of diffusion equation

$$
\frac{d \zeta}{d t}-D \nabla^{2} \zeta=\eta
$$

The equation retains some of the stochastic character of the original system through the noisy source term $\eta \cdot \eta$ is a noise term, satisfying $\langle\eta\rangle=0$, and

$$
<\eta(x, t) \eta\left(x^{\prime}, t^{\prime}\right)>=2 R \delta\left(t-t^{\prime}\right) \delta\left(x-x^{\prime}\right)
$$

Here $R$ is a function describing the local reaction rate at $x$, the number of reactions (deals) per unit length per unit time, which has been shown [30] to have the scaling form $R(x)=(J / D) S(x / w)$, where $w$ is the width of the reaction front. After integrating $R$ over $x$ one has $\int d x R(x)=J$. We seek solutions to this equation with constant flux on the boundaries, whose noise-averaged value is zero at the center of the front. We enforce the condition that the noise-averaged slope at the boundaries is $\sim-J / D$, so that the inward flux of buyers and sellers at the boundaries is maintained at $J$. We solve this equation on the closed interval $[-L / 2, L / 2] \subset \mathcal{R}$, which here plays the role of the price space $\mathcal{P}$. We write the most general solution as an expansion in Fourier modes

$$
\zeta(x, t)=-(J / D) x+\sum_{n=0} \chi_{n}(t) \cos \left(\frac{(2 n+1) \pi x}{L}\right)
$$


where the $\chi_{n}$ are the time dependent, noise dependent Fourier coefficients.

$$
\chi_{n}(t)=\frac{2}{L} \int_{0}^{t} d t^{\prime} \int d x^{\prime} \eta\left(x^{\prime}, t^{\prime}\right) \cos \left(\frac{(2 n+1) \pi x^{\prime}}{L}\right) \exp \left[\frac{(2 n+1)^{2} \pi^{2} D}{L^{2}}\left(t^{\prime}-t\right)\right]
$$

Modes $n$ with wavelengths $\frac{L}{(2 n+1)}<w$ oscillate many times within the reaction front, and therefore do not contribute to the sum (3.25). We may therefore simplify the calculation by approximating the noise source $\eta$, distributed by the sharply peaked envelope function $R$, as a point source at the origin,

$$
\begin{gathered}
\eta(t)=\frac{1}{\sqrt{2 J}} \int d x^{\prime} \eta\left(x^{\prime}, t^{\prime}\right) d x^{\prime} \\
<\eta(t) \eta\left(t^{\prime}\right)>=\delta\left(t-t^{\prime}\right) \frac{1}{2 J} \int d x R(x)=\delta\left(t-t^{\prime}\right)
\end{gathered}
$$

and restricting the sum over $n$ to $0<n<N_{\max }=\frac{L}{w}$.

The quantity $\zeta(x, t)$ represents the difference between the density of buyers and sellers at the point $x$, at time $t$. This is not directly one of the quantities we are interested in, but it is related. The zero point of $\zeta$ represents the center of the market, the midmarket $M(t), \zeta(M(t), t)=0$. Since the density difference goes through its zero point linearly with slope $\sim-J / D$, its fluctuations at the point $x=0$ are proportional to the fluctuations of the position of the zero of $\zeta$, i.e. the midmarket. Thus, the midmarket position $M(t)$ may be written (neglecting terms of order $<M^{2}>/ L^{2}<<1$ )

$$
M(t)-\bar{M}=\frac{D}{J} \zeta(0, t)=\frac{D}{J} \sum_{n=0}^{N_{\max }} \chi_{n}(t)
$$

where we have allowed for the possibility $\bar{M}=<M>\neq 0$ even though $<M>=0$ in (3.25). The noise averaged value of $M(t)-\bar{M}$ is 0 , but the noise dependent terms cause it to fluctuate, and we may calculate the magnitude of these fluctuations

$$
w^{2}=\frac{D^{2}}{J^{2}}\left(<\zeta^{2}(0, t)>-<\zeta(0, t)>^{2}\right)=\frac{D^{2}}{J^{2}} \sum_{n}^{N_{\max }} \sum_{m}^{N_{\max }}<\chi_{n}(t) \chi_{m}(t)>
$$

as well as the autocorrelation function $W(T)=<M(t) M(t+T)>-<M(t)><$ $M(t+T)>$

$$
W(T)=\frac{D^{2}}{J^{2}}(<\zeta(0, t) \zeta(0, t+T)>-<\zeta(0, t)><\zeta(0, t+T)>)
$$

$\S$ The dependence on $w$ of our approximate expectation will lead to a self-consistent definition for $w$, and a logarithmic correction the dimensional analysis of the previous section 


$$
=\frac{D^{2}}{J^{2}} \sum_{n}^{N_{\max }} \sum_{m}^{N_{\max }}<\chi_{n}(t+T) \chi_{m}(t)>
$$

The angle brackets mean average over the noise $\eta$, invoking the expression (3.28) above. Using (3.25) - (3.28) it is easy to see that for large $t$

$$
<\chi_{n}(t) \chi_{m}(t)>=\frac{8 J}{\pi^{2} D} \frac{1}{(2 n+1)^{2}+(2 m+1)^{2}}
$$

and one gets 29]

$$
w=\left[\frac{\ln (c L / w)}{\pi(J / D)}\right]^{1 / 2}
$$

where the logarithmic factor is due to the summation $\sum_{n, m}\left[(2 n+1)^{2}+(2 m+1)^{2}\right]^{-1}$ over $n$ and $m$ from 1 to $N_{\max }=L / w$. As expected from dimensional analysis, the width of the reaction front goes like $\sqrt{D / J}$. Note also that the midmarket variance grows, slowly, with $L$. This is an example of the logarithmic corrections to our crude dimensional analysis of the scaling laws mentioned in section 2. It is interesting because it implies that having broad masses of traders behind the best bid and offer will have a (weak) influence on the behavior of the midmarket, increasing its variance - presumably other scaling laws will be similarly affected. Thus, choosing the parameter $L$ in our model is not an arbitrary choice, or technical problem. $L$ defines how tightly traders are bunched together in price space outside of the reaction front, and is therefore a parameter carrying meaningful dynamical information about the market, which we may measure along with the other parameters of the market, $J$ and $D$.

Finally we note that the width (3.33) does not widen with time, which makes sense in a stable market, not subjected to external shocks. We shall see in the next section how these shocks actually may be added to our model, resulting in the usual result for Brownian motions of a variance growing linearly in time.

We may calculate the correlator (3.31) by the same method as the width. The result is

$$
W(T)=\frac{2 D}{J \pi^{2}} \int \frac{d x d y}{x^{2}+y^{2}} e^{-x^{2} J|T|} \approx \frac{D}{J \pi}\left(E_{1}(J|T|)-E_{1}\left(w^{2} J|T| / L^{2}\right)\right)
$$

where the integration is from $\epsilon=\sqrt{D / J L^{2}}<<1$ to 1 and $E_{1}(x)$ is the exponential integral $E_{1}(x)=\int_{x}^{\infty} \frac{e^{-t} d t}{t}$. For small $x E_{1}(x) \sim-\ln x$ and for large $x$ it is exponentially 
small. There are three time intervals: $T<1 / J, 1 / J<T<1 / J \epsilon^{2}$ and $T>1 / J \epsilon^{2}$. In the first interval $x^{2} J|T|<1$ for all $x$ and we can expand $e^{-x^{2} J|T|} \approx 1-x^{2} J|T|$ which gives us $W(T)=w^{2}-(1 / 2 \pi) D T$. In the interval $1 / J<T<1 / \epsilon^{2} J$ one can neglect $E_{1}(J|T|) \sim e(-J T)$ and only $E_{1}\left(\epsilon^{2} J|T|\right)$ is important, so

$$
W(T) \approx \frac{D}{2 \pi J} \ln \left[\frac{L^{2}}{w^{2} J T}\right], \quad 1<J T<1 / \epsilon^{2}
$$

In the third interval the correlation function is exponentially small $W(T) \sim e\left(-\left(w^{2} J|T| / L^{2}\right)\right)$ - but $T>1 / \epsilon^{2} J$ represents time scales so long as to be beyond the scope of this model.

Note that, because the correlation function is a function of the time difference $T$ a measurement of $W(T)$ for a range of values $T$ contains enough information to fix all of the unknown parameters in the minimal model.

\section{Nonstationary Markets: the Minimal Model with Asymmetric Fluxes}

It is also possible to consider the minimal model with different fluxes for the buyers and the sellers. Cardy et.al. scheme may be used with few modifications. The principal difference is the non-stochastic part of the solution $\zeta(x, t)$ must be time dependent, and satisfy the appropriate slope conditions at the boundaries. Let $\bar{J} \pm \Delta J$ be the flux at the upper (lower) boundary. Then the most general solution for $\zeta$ is now

$$
\zeta(x, t)=-\frac{\bar{J}}{D} x-\frac{\Delta J}{2 L D} x^{2}-\frac{\Delta J}{L} t+\sum_{n} \chi_{n}(t) \cos \left(\frac{(2 n+1) \pi x}{L}\right)
$$

The nonstochastic part no longer vanishes at $x=0$ - instead its zero is a function of time $x_{0}(t)$. For small times

$$
x_{0}(t) \sim \frac{-2 D \Delta J}{\bar{J} L} t
$$

and this defines the speed of the moving midmarket. Following the same logic as before we must then evaluate

$$
w^{2}=\frac{D^{2}}{\bar{J}^{2}}\left(<\zeta^{2}\left(x_{0}(t), t\right)>-<\zeta\left(x_{0}(t), t\right)>^{2}\right) .
$$

which leads to the result

$$
w_{\Delta J}^{2}(t)=\frac{2 D}{\pi^{2} \bar{J}} \int \frac{d x d y}{x^{2}+y^{2}} \cos (\nu x) \cos (\nu y)
$$


where $\nu=(2 / \pi)(\Delta J / \bar{J})(\bar{J} t) \epsilon$ Now $w_{\Delta J}$ explicitly depends on $t$ and because $\cos (\nu x)<1$ the width is smaller and decreasing with time. For short times, this may be expanded out as

$$
w_{\Delta J}^{2}(t)=w^{2}-\frac{2 D}{\pi^{2} \bar{J}} \nu^{2}
$$

The correlation function may be calculated as well. The result is

$$
W_{\Delta J}\left(t, t^{\prime}\right)=\frac{\bar{J}}{D} \int \frac{d x d y}{x^{2}+y^{2}} e^{-x^{2} \bar{J}\left|t-t^{\prime}\right|} \cos (\nu x) \cos \left(\nu^{\prime} y\right)
$$

and now it is not only a function of $T=t-t^{\prime}$, but also of $t$ and $t^{\prime}$ separately due to $\cos (\nu x)$ and $\cos \left(\nu^{\prime} y\right)$ factors. One can again study the behaviour of the correlation function in detail as before, and we do so in the next section.

\section{Beyond the Minimal Model}

\section{Markets Under the Influence of Random Disturbances}

As we discussed in the introduction, it is possible to consider the minimal model with the additional feature of random disturbances applied. This will have the effect of injecting "energy" into the market, causing the system to be partly in the low lying excited states. Our approach to calculating this sort of effect will be to introduce an external source of noise into one of the parameters of the model, and average our calculations over that noise source. Implicit within this procedure is the assumption that these fluctuations occur over time scales much shorter than those of measurement. We may imagine several kinds of disturbances.

\section{Random Fluctuations in Trader Drift}

The first might be considered a disturbance due to the influence of news. A market's internal structure is buffeted throughout the day by news announcements, and the behavior of markets for related assets. To model this effect and observe its effect on the scaling of market microstructure, we subject our diffusing traders to a random white-noise drift $v(t)$, proportional in strength to the trade price $<v(t) v\left(t^{\prime}\right)>=S T_{d} \delta\left(t-t^{\prime}\right)$, where $S$ is the approximate price at the midmarket, and $T_{d}$ is a parameter which describes the 
strength of the noise. This noise is intended to be the response of traders to a series of small news items, both good and bad. The effect of this on the stable market represented by the minimal model is to cause the trade prices to execute a random walk. We then modify the equation for $\zeta$ as

$$
\frac{d \zeta}{d t}-D \nabla^{2} \zeta=\eta+v(t) \frac{\partial \zeta}{\partial x}
$$

We can solve this equation by perturbation in $v(t)$, so that $\zeta(x, t)=\zeta_{0}(x, t)+\zeta_{1}(x, t)+\ldots$, $\zeta_{0}(x, t)$ is a solution to $(3.25)$ and

$$
\frac{d \zeta_{n}}{d t}-D \nabla^{2} \zeta_{n}=\eta+v(t) \frac{\partial \zeta_{n-1}}{\partial x}
$$

Because we are interested in finding $\zeta(x, t)$ at small $x$ it is easy to see from

$$
\frac{\partial \zeta_{0}}{\partial x}=-(J / D)-\sum_{n=0} \frac{(2 n+1) \pi}{L} \chi_{n}(t) \sin \left(\frac{(2 n+1) \pi x}{L}\right)
$$

that one can neglect all $\chi_{n}(t)$ terms, because they are suppressed as $x / L$, so $\zeta_{1}(x, t)=$ $-(J / D) \int d t v(t)$ and $\partial_{x} \zeta_{1}=0$, which means that the solution in the presence of drift is

$$
\zeta(x, t) \approx-(J / D) x+\sum_{n=0} \chi_{n}(t) \cos \left(\frac{(2 n+1) \pi x}{L}\right)-(J / D) \int d t v(t) .
$$

We see that the effect on the solution $\zeta$ is only to add a Brownian motion to the solution, independent of $x$. This leads to the following results for the reaction front width $w^{2}=<$ $M^{2}>-<M>^{2}$ and correlation function $W\left(t-t^{\prime}\right)=<M(t) M\left(t^{\prime}\right)>-<M(t)><$ $M\left(t^{\prime}\right)>$ for the MidMarket field $M(t)=(D / J) \zeta(0, t)$

$$
\begin{array}{r}
w^{2}(t)=\frac{D}{\pi J} \ln (1 / \epsilon)+S T_{d}\left(t-t_{0}\right) \\
W\left(t, t^{\prime}\right)=W_{0}\left(t-t^{\prime}\right)+S T_{d} \min \left(\left(t-t_{0}\right),\left(t^{\prime}-t_{0}\right)\right)
\end{array}
$$

and $t_{0}$ is the moment when the drift $v(t)$ was switched on. The fluctuations in $M(t)$ start out as a constant, but then after the characteristic time $\frac{D}{J S T_{d}}$, they grow in time, as expected for a Brownian motion. This is consistent, both with standard option theory, and with the common observation that intra-day volatilities are larger than expected for a Brownian motion. Furthermore, we see that the parameter $T_{d}$ should be identified with the volatility of the trade price. 


\section{Random Fluctuations in the Flux of New Traders}

The second type of noise to which we subject the system is a fluctuating rate of traders entering the market. It is obviously somewhat more general to assume a randomly fluctuating entry rate than a constant one, and so, at the expense of introducing an additional parameter, we nudge our model one step closer to a realistic market. Following Cardy, we may once again compute mean field theoretic approximations to some of the market microstructure. This calculation may be done directly from the results of the previous section, for a market with unequal trader influxes, by taking the quantity $\Delta J$ to be randomly fluctuating $<\Delta J(t)>=0,<\Delta J(t) \Delta J\left(t^{\prime}\right)>=\mu^{2} \bar{J}^{2} F\left(t-t^{\prime}\right), \quad F(0)=1$. Note that we must have $\mu<<1$ in order to ensure that $J$ is always positive. Using (3.39) we find

$$
w_{\Delta J}^{2}(t)=<\frac{2 D}{\pi^{2} \bar{J}} \int \frac{d x d y}{x^{2}+y^{2}} \cos (\nu x) \cos (\nu y)>
$$

We calculate the average using the relation

$$
<\cos (\nu x) \cos (\nu y)>=1 / 2\left[\exp \left(-<\nu^{2}>(x+y)^{2} / 2\right)+\exp \left(-<\nu^{2}>(x-y)^{2} / 2\right)\right]
$$

and making use of $\nu=(2 / \pi)(\Delta J / \bar{J})(\bar{J} t) \epsilon$ we have $\left\langle\nu^{2}>=(2 \bar{J} \epsilon / \pi)^{2} \mu^{2} t^{2}\right.$. Expanding in $<\nu^{2}>$ we have for small $t$

$$
\begin{array}{r}
w_{\Delta J}^{2}(t) \approx \frac{2 D}{\pi^{2} \bar{J}} \int \frac{d x d y}{x^{2}+y^{2}}\left[1-\frac{1}{2}<\nu^{2}>\left(x^{2}+y^{2}\right)\right] \\
=\frac{D}{\pi \bar{J}}\left[\ln (1 / \epsilon)-\frac{(2 \epsilon \mu \bar{J} t)^{2}}{\pi^{3}}\right]
\end{array}
$$

and we see by solving (4.8) that the correction to the total fluctuation width due to random noise in $J$ is negative, and quadratic in time.

\section{Random Fluctuations in Trader Volatility}

Finally, we consider noise added to the trader volatility $D$, and again we may calculate its effects in mean field theory. To this end, we introduce a small noisy term $d(t)$ to be added onto the average diffusion constant $\bar{D}, D=\bar{D}+d(t)$, where $\langle d(t)\rangle=0$ and $<d(t) d\left(t^{\prime}\right)>=T_{D} \delta\left(t-t^{\prime}\right)$, and solve the equation

$$
\frac{d \zeta}{d t}-D \nabla^{2} \zeta=\eta+d(t) \nabla^{2} \zeta
$$

perturbatively in $d(t): \zeta=\zeta_{0}+\zeta_{1}+\ldots . T_{D}$ must be very small $T_{D}<<\bar{D}^{2}$, so that the total trader volatility $D$ is always positive. The equation for $\zeta_{1}$ is

$$
\frac{d \zeta_{1}}{d t}-\bar{D} \nabla^{2} \zeta_{1}=d(t) \nabla^{2} \zeta_{0}
$$




$$
=-d(t) \sum_{n} \chi_{n}(t)\left(\frac{(2 n+1) \pi}{L}\right)^{2} \cos \left(\frac{(2 n+1) \pi x}{L}\right)
$$

and

$$
\zeta_{1}(x, t)=\sum_{n=0} \chi_{n}^{1}(t) \cos \left(\frac{(2 n+1) \pi x}{L}\right)
$$

where $\chi_{n}^{1}$ is easily found to be

$$
\chi_{n}^{1}(t)=-\int_{0}^{t} d t^{\prime} d\left(t^{\prime}\right) \chi_{n}\left(t^{\prime}\right)\left(\frac{(2 n+1) \pi}{L}\right)^{2} \exp \left[\frac{(2 n+1)^{2} \pi^{2} \bar{D}}{L^{2}}\left(t^{\prime}-t\right)\right]
$$

and $\chi_{n}\left(t^{\prime}\right)$ is given by (3.26). It is easy to see that $\left\langle\zeta_{0}(t) \zeta_{1}(t)\right\rangle=0$ because $\zeta_{0}(t)$ does not depend on the noise $d(t)$ and $\zeta_{1}(t)$ is linear in $d(t)$ so $<\zeta_{0}(t) \zeta_{1}(t)>\sim<d(t)>=0$. Then $\left\langle\zeta^{2}(t)>=<\zeta_{0}^{2}(0, t)>+<\zeta_{1}^{2}(0, t)>\right.$ and the midmarket fluctuation width is given by

$$
w^{2}=<M^{2}(t)>=\frac{D^{2}}{J^{2}} \sum_{n, m}\left[<\chi_{n}(t) \chi_{m}(t)>+<\chi_{n}^{1}(t) \chi_{m}^{1}(t)>\right]
$$

We have already calculated $<\chi_{n}(t) \chi_{m}(t)>(3.32)$ and using the same method it is easy to calculate

$$
\begin{gathered}
<\chi_{n}^{1}(t) \chi_{m}^{1}(t)>=T_{D} \frac{\pi^{2}}{L^{2} \bar{D}} \frac{(2 n+1)^{2}(2 m+1)^{2}}{(2 n+1)^{2}+(2 m+1)^{2}}<\chi_{n}(t) \chi_{m}(t)> \\
=T_{D} \frac{8 J}{L^{2} \bar{D}^{2}} \frac{(2 n+1)^{2}(2 m+1)^{2}}{\left[(2 n+1)^{2}+(2 m+1)^{2}\right]^{2}}
\end{gathered}
$$

After summation over $n$ and $m$ we get an extra factor $N_{\max }^{2}=L^{2} / w^{2}$ and the width

$$
w^{2}=\frac{\bar{D}}{\pi J} \ln (1 / \epsilon)+\frac{\pi}{4} \frac{T_{D}}{J} \frac{1}{w^{2}}
$$

and we see that the correction scales as $1 / J w^{2}$ for small $T_{D}$, and is independent of time. When $T_{D}$ gets larger the scaling law for $w^{2}$ is going to change - it is clear that for $J>\bar{D}^{2} / T_{D}$ the second term is bigger than the first one and one might conclude that $w \sim J^{-1 / 4}$. However, $T_{D}$ cannot be too large, because otherwise the total trader volatility $D=\bar{D}+d(t)$ can go negative.

In conclusion let us note that the three different fluctuations in the market parameters lead to three different types of corrections to the width: fluctuations in $J, D$, and the 
trader drift parameter each lead to corrections to the width. Noise in the drift leads to a positive correction to $w^{2}$, growing linearly in $t$, and independent of $J$. Noise in $J$ leads to a negative correction, growing quadratically in time, and noise in $D$ leads to a positive correction independent of time. The corrections to the autocorrelation function are also calculable, and are interesting because the function contains more information, which we may use to extract the parameter of $D, J$, and $L$ precisely, and to provide additional tests of these conjectures.

\section{Two-Liquid Model}

During a crash, it is clear that a market behaves altogether differently from the quiescent markets on which our efforts have so far been concentrated. It is known, for example, that bid-offer spreads widen out hugely. Nevertheless, it is reasonable to expect a market in this state to exhibit some sort of scaling behavior. In this picture, the crashing market is a different phase, as a gas phase is different from a liquid phase. A model for this phase would have to have a different ground state, one which allowed for this sort of crashing dynamics.

The simplest model for a market crash was suggested at the end of the minimal model discussion, in which a mean field calculation was done for some of the market microstructure when buyers and sellers entered the market at unequal rates. However, this does not cause spreads to widen out appreciably, and there is a good reason why. Best bids and offers can only be calculated from the bids and offers of limit-order traders, who submit specific prices at which they will trade. During a crash, a large number of trades are executed as market orders, that is, with no prior warning of the position in price space that that trader had occupied. We thus introduce a second type of buyer and seller into the market, the market order trader, who is distinguished from the limit order trader only in that we cannot see his bids and offers on screen. Thus, the best bid and offer continue to be calculated from the limit order traders, even though most of the trading volume is now being conducted by market order sellers, with limit order buyers.

This model can be formulated as one in which there are two flavors of buyer, $B$ and $B^{\prime}$, and two flavors of seller $S$ and $S^{\prime} . B$ and $S$ are limit order traders and $B^{\prime}$ and $S^{\prime}$ are market order traders. The prices of the unprimed traders only are used to calculate the best bid and offer on the trade screen, and are therefore visible to other traders. Thus the two-fluid model includes the reactions $B+S \longrightarrow 0, B^{\prime}+S \longrightarrow 0, B+S^{\prime} \longrightarrow 0$, but not $B^{\prime}+S^{\prime} \longrightarrow 0$, because these primed traders are invisible to each other. It is easy to write an evolution operator like (3.18) for all 4 fields $\Psi_{b}, \Psi_{b^{\prime}}, \Psi_{s}$ and $\Psi_{s^{\prime}}$ which 
describes the diffusion of all 4 fields and annihilation interactions between them of the form $\lambda\left(\bar{\Psi}_{i} \bar{\Psi}_{j}-1\right) \Psi_{i} \Psi_{j}$ for three possible pairs $(i, j)=(b, s),\left(b^{\prime}, s\right)$ and $\left(b, s^{\prime}\right)$. There is no pair $\left(b^{\prime}, s^{\prime}\right)$ - market order traders do not see each other.

For low density and flux of the market order traders, the dynamics of this model is expected to be dominated by limit order traders, and is essentially the same as the minimal model. Consider now the case where the density and flux for market order traders equals that of the limit order traders. In this case there are very few dimension-ful parameters, so dimensional analysis regains some predictive power. We expect scaling laws exactly like those of the minimal model, in particular the spread should scale like $\operatorname{Spr} \sim \sqrt{(D / J)}$. However, market order traders cause the spread to widen out, because they trade only with limit order traders, pushing the spread farther out each time they do. This effect was discussed in [8]. Thus, we expect a numerical prefactor, greater than 1, relating the minimal model spread to that of the two-liquid model at this symmetric point.

When the density and flux of market order traders dominate that of the limit order traders, we expect a very wide spread, as a result of the market order traders pushing limit order bids and offers far away from each other. In this situation the market order best bid $B^{\prime}$ is very near to the limit order best offer $O: B_{t}^{\prime} \sim O_{t}$, and is far above the market order best offer, which is near to the limit order best bid: $O_{t}^{\prime} \sim B_{t}$, in the fashion of an across market, because they cannot see one another. In this case, the limit order traders no longer trade with one another, because they are so far away, and we have two decoupled minimal model systems, the $\left(b^{\prime}, s\right)$ system and the $\left(b, s^{\prime}\right)$ system. The averaged spread in this case grows linearly with time, with each side moving at the rate determined by the formula (3.37) from the previous section. In practice, as discussed by [8], this unstable situation stabilizes itself when market order traders switch themselves to limit order traders, but that is beyond the scope of this model. Finally, in a crash situation, we expect an asymmetric flux of buyers and sellers, with most sellers being market order sellers. Again the $\left(b^{\prime}, s\right)$ and the $\left(b, s^{\prime}\right)$ systems are decoupled into two minimal models, and the averaged spread again grows linearly with time, as in formula (3.37). In this situation, which is the situation of interest in this model, market order sellers cannot switch to limit order sellers and reasonably expect to get their order executed, so this result is expected to hold.

This model is much harder to treat analytically - method of Cardy and collaborators [29 may be used, but none of the quantities accessible to us are interesting. We have 4 fields instead of 2 and many more free parameters (different fluxes for limit and market 
order traders, etc.). In the mean field approximation, neglecting the quartic term, one gets for the densities $\rho_{i}(x, t)=<\Psi_{i}(x, t)>$

$$
\begin{aligned}
& \left(\partial_{t}-D \partial_{x}^{2}\right) \rho_{B}+\lambda \rho_{B}\left(\rho_{S}+\rho_{S^{\prime}}\right)=0 ; \quad\left(\partial_{t}-D^{\prime} \partial_{x}^{2}\right) \rho_{B^{\prime}}+\lambda \rho_{B^{\prime}} \rho_{S}=0 \\
& \left(\partial_{t}-D \partial_{x}^{2}\right) \rho_{S}+\lambda \rho_{S}\left(\rho_{B}+\rho_{B^{\prime}}\right)=0 ; \quad\left(\partial_{t}-D^{\prime} \partial_{x}^{2}\right) \rho_{S^{\prime}}+\lambda \rho_{S^{\prime}}\left(\rho_{B}=0\right.
\end{aligned}
$$

and if all diffusion coefficients are the same $D=D^{\prime}$ and one finds a particularly simple equation for the quantity $\zeta(x, t)=\rho_{B}(x, t)+\rho_{B^{\prime}}(x, t)-\rho_{S}(x, t)-\rho_{S^{\prime}}(x, t)$, which may be interpreted as the density difference of all buyers and sellers.

$$
\frac{d \zeta}{d t}-D \nabla^{2} \zeta=0
$$

This is the same mean field equation as in the minimal model but unfortunately the non-linear effects cannot be incorporated through a single noise term. Furthermore, the zero of $\zeta$ cannot be interpreted as the midmarket. We are thus confined to numerical simulations for this model, at least for now. We shall report on computer simulations of this "Two Fluid Model" in the crashing phase, in an upcoming publication. It will be interesting to see if in the crashing phase this model leads to log-periodic oscillations 42.

\section{The Bias Model}

As mentioned in the introduction, the bias model is a modification of the minimal model introduced above, in which the diffusion operators $D_{B}$ and $D_{S}$ are modified to contain a drift, which increases when the last trade has ticked upwards, and decreases when the last trade has ticked downward. (The amount by which it increases or decreases is controlled by a parameter which we refer to as the "market tension".) This means that our state, our probability distribution at time $t$, now depends not only on the positions of traders, but also on the drift.

This model is designed to develop instabilities, and numerical simulations show that, when the drift parameter $\epsilon$ reaches a critical value $\epsilon_{\text {crit }}$, the model does indeed crash, either upwards or downwards. It has the interesting feature that, somewhat like phase transitions in physical materials, or in traffic patterns, the approach to this transition is characterized by interludes during which the behavior of the system crosses over to the new (crashing) behavior, which increase in duration as $\epsilon$ approaches $\epsilon_{\text {crit }}$. The phase transition to this new behavior occurs because the drift velocity has grown larger than the diffusive velocity, and begins to dominate the market's movements. Dimensional analysis suggests that $\epsilon_{\text {crit }} \sim J^{1 / 2}$, which makes sense - crashes are more common in thin markets than in liquid ones. 
The instability in this model is analogous to a particle rolling off of a sphere, in which the crash may take an arbitrarily large time to develop. Although any positive bias eventually leads to a crash, we regard as unstable only those models which lead to a crash within the prediction horizon of our model, which can be no longer than a few hours, and certainly cannot extend past the market close.

There are several interesting problems to attack. The first is the new, crashing behavior itself - we would like to understand how a market's behavior is altered during such a crash. The simplest way to do this is to measure the liquidity scaling laws during the crash, and see how they are changed. Dimensional analysis suggests that they should be modified, because the drift $\epsilon$ itself is a new dimension-ful parameter whose presence now allows a much wider variety of behavior. In such a phase, market order traders would be expected to play a large role and thus should be included in any simulation, as in the Two-Fluid Model (see the previous section).

An even more interesting problem would be how the market's behavior is modified in the prelude to a crash. Again, liquidity scaling laws would be the simplest quantity to measure in such a case, and again dimensional analysis suggests that they should be modified appreciably in the prelude to a crash, within the framework of our model. It is plausible that momentum-trading is what becomes important at such moments, and were such a conjecture born out by empirical tests in the market, its utility would be obvious.

In addition to the liquidity scaling laws, there is the behavior of the drift $\epsilon$ itself. In this simple model, drift feeds on itself. The parameter $\epsilon$ sits inside a meta-stable basin for a while, but is buffeted back and forth by chance movements in the market, until it is knocked out of its basin, and into the unstable region near $\epsilon_{\text {crit }}$. Once here, it gathers momentum under its own steam. Its trajectory in the unstable region would be an interesting quantity to calculate.

The bias model describes the onset of crashes, but not their eventual petering out, because in this model traders remain in the drift-dominated crashing mode until there are no traders left. An interesting extension of this model would be one in which drift is somehow dynamically restored to small values, allowing the market to return to the quiescent state. A model of this kind would enable us to estimate the magnitude and duration of crashes, because with this new feature they are now only of finite size.

\section{Market Makers}

The notion of diffusing traders, who change their trade prices often before trading, 
may be considered also in the context of a market with a single monopolistic market maker, or even several competing market makers. This problems has been studied in great detail, from the point of view of inventory [3, 4, 5, 6, 11, 0, 21], strategic traders [10], and in the presence of information imbalance 9].

We do not intend to review all this work here, we merely suggest that in the types of markets alluded to in the introduction, dominated by day-trading traders, the modeling of the order arrival process might be modified. More specifically we propose to refine the modeling of the order arrival process by using the information contained in the book of limit orders, together with the diffusion proposal. To this end, we demonstrate this calculation in a toy model, which omits all the other effects mentioned above, inventory, trader strategy, and information imbalance.

We imagine a market maker who is allowed to see every limit order available in the market. As a first approximation, we assume that only limit order traders are present in the market. The market maker may set his bid and offer anywhere he likes. He desires to maximize his profit, while minimizing the possibility of any open position. The market maker will set his constant bid and offer optimally for a time horizon $T_{H}$. We may define, during this horizon, the expected number of shares he will sell, as

$$
\Phi_{S}=\int_{t_{0}}^{T_{H}} d t \int_{L_{B}}^{\infty} d x^{\prime} G^{\prime}\left(L_{B}, x^{\prime} ; t, t_{0}\right) \rho_{S}\left(x^{\prime}, t_{0}\right)
$$

where $G\left(x, y ; t, t^{\prime}\right)$ is the Green's function for the diffusion operator, which satisfies Dirichlet $G=0$ boundary conditions at $x=L$. Similarly the expected number of shares he will buy is

$$
\Phi_{B}=\int_{t_{0}}^{T_{H}} d t \int_{0}^{L_{S}} d x^{\prime} G^{\prime}\left(L_{S}, x^{\prime} ; t, t_{0}\right) \rho_{B}\left(x^{\prime}, t_{0}\right)
$$

In terms of this quantities, his expected profit is

$$
P_{N}=\left(L_{S}-L_{B}\right) \min \left(\Phi_{B}, \Phi_{S}\right)
$$

and his expected open position is

$$
P_{O}=\left|\Phi_{B}-\Phi_{S}\right|
$$

Thus, we would like to maximize $P_{N}$ while enforcing $P_{O}=0$. We may formulate this as a minimization problem using the technique of Lagrange multipliers, as $L_{S}, L_{B}, \lambda$ such that

$$
F=P_{N}-\lambda P_{O}
$$


is minimized However, it is more simply reformulated as $L_{S}, L_{B}, \lambda$ such that

$$
\tilde{F}=L_{B} \Phi_{S}-L_{S} \Phi_{B}-\lambda\left(\Phi_{B}-\Phi_{S}\right)
$$

is minimized, because the functionals $F$ and $\tilde{F}$ have the same minima. Minimization of $\tilde{F}$ leads to the equations

$$
\begin{gathered}
\Phi_{S}+\left(L_{B}+\lambda\right) \frac{\partial}{\partial L_{B}} \Phi_{S}=0 \\
\Phi_{B}+\left(L_{S}+\lambda\right) \frac{\partial}{\partial L_{S}} \Phi_{B}=0 \\
\Phi_{B}=\Phi_{S}
\end{gathered}
$$

These equations require numerical solution.

\section{Towards Experimental Tests of These Conjectures.}

At the heart of this paper is a proposal that experiments be carried out, testing the results obtained above and in future publications, regarding scaling laws in market microstructure, for the markets in which we are interested. We describe here what is intended, and will report on work in this direction in a future publication 35].

The experimental tests of the above results may be done by testing against real market data, over the short time scales necessary to determine the elementary statistical fields $B, O, \chi$, and their correlations.

Having stated several interesting conjectures in the previous sections, we desire to test them experimentally. This appears to pose some difficulty - it is not immediate that any of these experiments can be done.

The reason is that the expectations in the above model are ensemble averages. That is, they are averages in which the same market is started in the same state many times over, and allowed to probabilistically evolve. The average is then taken over all the different possible "worlds" that these different probabilistic paths may take. However, in the real 
world, there is only one path. We do not have the ability to sample many different worlds. The only averaging we can do is time-averaging, which in principle is quite a different thing.

However, we can show by a simple argument that, for certain well-behaved quantities, they are in fact, the same thing. This occurs because, for these quantities, the system is "forgetful", so that the system, after a while, has reset itself, and after the forgetting time $T$, is actually the same as another version of the initial world. In other words, after $N$ forgetting times $T$, we have actually averaged over $N$ ensemble members.

We show this by considering the ensemble fluctuations of the time average of an operator, and show that they go to zero, under certain conditions. This means that the operator is diagonal on any state, i.e. a constant times the identity matrix, and in that case it's ensemble average must be equal to the constant. By time translation invariance, this constant is also equal to the expectation of the (un-time-averaged) operator.

Notice that we require time translation invariance, here. If the state is not at least approximately in a steady state, then the theorem is just plain false. However, no one would expect to get a good experimental measurement, either, of any of the elementary statistical fields, if the state were to sharply change it's character during the measurement. Thus a steady state, or a quasi-steady-state, is a necessary precondition for both measurement of a time-averaged quantity and comparison of that quantity to the ensemble averages we propose to calculate.

The time average of an operator $O(t)$ is $(1 / T) \int_{t}^{t+T} O(\tau) d \tau$. Notice that it is still an operator, and therefore contains information about all the different possible worlds defined by the different paths the world might take. But as $T \longrightarrow \infty$ this operator often becomes a scalar times the identity matrix. To see this, we compute it's fluctuations in some time translation invariant state.

$$
\begin{gathered}
\operatorname{Fluc}(\bar{O})=<\left(\frac{1}{T} \int_{t}^{t+T} d \tau O(\tau)-<\frac{1}{T} \int_{t}^{t+T} d \tau O(\tau)>\right)^{2}> \\
=<\int_{t}^{t+T} d \tau \int_{t}^{t+T} d \tau^{\prime} O(\tau) O\left(\tau^{\prime}\right)>-<\int_{t}^{t+T} d \tau O(\tau)>^{2} \\
=\int_{t}^{t+T} d \tau \int_{t}^{t+T} d \tau^{\prime} \operatorname{Corr}\left(O(\tau) O\left(\tau^{\prime}\right)\right)
\end{gathered}
$$

Thus we see that, if the correlation of $O$ at different times falls off with $T$, the time average will also fall off to zero, as $T$ gets large. Hence we arrive at the condition that the 
only quantities we may compare with experiment are those whose time-time correlation functions decays with time $T$. Fortunately, this is true of most such quantities - indeed their correlations usually decay exponentially.

Using this result, we may propose a method of measuring the mooted scaling laws. We use the bid-offer spread as an example to illustrate the method. We record the bid-offer spread, and traded volume at every instant of time (with a time spacing small enough to catch every movement of bid and offer prices). We find an averaging time sufficient to satisfy the time-averaging relation above, and compute from this recorded data a series of time averages of spread versus deal rate. We then make a scatter plot of $\log S$ versus $\log J$, and compute a best fit line to this scatter plot - the prediction of the minimal model is that this slope should be $-1 / 2$.

The bid-offer spread in dealer markets, as opposed to the double auction of this paper, has been studied empirically by 13, 14, 16, 15, 22, 0, 23, 19, 21, 20, 25. These studies were motivated by debates on market design. For example, the restrictions on trading of the registered traders in the Toronto Stock Exchange (TSE), compared against the affirmative obligations for members of the New York Stock Exchange (NYSE), and of the more independent dealers of the OTC was studied in [16]. The behavior of the spreads of the specialists on the NASDAQ vs. the dealers in the OTC was studied by [20], the spreads on the AMEX were compared with those of the NYSE by [25], and the issue of competitive dealing on the NYSE was studied by [15, 21]. This field of study was in fact originated by Demsetz, who studied dealer spreads on the NYSE[13]. Each of these studies consisted of a linear regression applied to a large number of markets in individual stocks, and showed (among other things) that spreads have a negative gradient with deal rate, as we expect in our markets. In the case of [13, 15, 21], this was used to demonstrated the industry-wide, but not firm-wide, scale economies that exist in the market for dealer serveices, and made the case against natural monopolies, in favor of competitive dealer services.

\section{Conclusion}

In this paper we have introduced into the study of market microstructure a series of models for markets made up of professional traders. These traders scrap for every basis 
point, and trade principally through limit orders, which they change frequently in response to the changing position of their book, their various perceptions of the direction of the market, and movements of different but related markets. This type of trader typically trades on behalf of an institution, such as a bank in, e.g. the interdealer broker markets. We modeled their changing limit orders as random walks which terminate when they collide with a price of the opposite kind, a buyer with a seller. This kind of dynamics, known as diffusion-annihilation dynamics, has been well-studied in the context of physics and chemistry.

We used this modeling framework to study the scaling of quantities related to market microstructure. Our expectation is that the purely statistical effects of having large numbers of traders in a market will be the dominant effect for these quantities, and we do not entertain the more difficult task of seeking the more subtle effects of trader behavior. Scaling laws quantify the coarsest features of the markets behavior, and do not require a detailed model. Hence we focused our efforts on these scaling laws.

Our scaling laws were evaluated as expectations in a measure. This measure was defined as the steady state solution of our dynamics, under the reasoning that a quiescent market, one not undergoing a large sudden movement, but only trading at an approximately constant rate, was like a steady state market. Furthermore, it is only in a steady state market that these scaling laws would be well-defined and meaningful.

We began by studying the scaling laws for various proxies for liquidity, such as the bidoffer spread Spr, time to midmarket trade $\tau$, trader density near the best bid/offer $\Xi_{(\cdot)}$, and midmarket variance $\xi$. We found $\operatorname{Spr} \sim J^{-1 / 2}, \tau \sim J^{-1}, \Xi_{(\cdot)} \sim J^{1 / 2}$, and $\xi \sim J^{-1 / 2}$. We then went on to study more complex correlations and conditional expectations as a function of $J$, whose qualitative behavior had a ready intuition. These included the correlation of time changes in best bid with time changes in best offer $C_{\Delta_{t} B \Delta_{t} O}$, the density at the best bid/offer conditioned on fluctuation in best bid/offer $\Xi_{(\cdot)}\left(\xi_{(\cdot)}\right)$, and the time change in the bid/offer spread conditioned on the bid/offer spread $\Delta_{t} \operatorname{Spr}(\mathrm{Spr})$. In the latter two cases we found the scaling forms $\Xi_{(\cdot)}\left(\xi_{(\cdot)}\right) \sim \sqrt{\frac{J}{D}} f\left(\frac{D}{J \xi_{(\cdot)}^{2}}\right)$, and $\Delta_{t} \operatorname{Spr}(\operatorname{Spr}=$ $\left.s_{0}\right) \sim \sqrt{\frac{D}{J}} f\left(\frac{D}{J s_{0}^{2}}\right)$.

We also examined response functions to density imbalance, estimated the size a tender offer must be to attract a certain number of buyers, and the expected profits of a specialist. Although we have, by dimensional analysis, extracted some information from our model, a full treatment of these scaling laws requires numerical simulation, the results of which 
shall appear in a forthcoming publication.

Some of our scaling laws were amenable to treatment by an approximation developed in [29]. We calculated the variance of the midmarket $w$, and found a logarithmic correction factor to our dimension-analytic result. The method also enabled us to calculate the time correlation function.

The above scaling laws and scaling forms were studied first in a model with as few parameters as possible, to isolate the purely statistical effects. We then introduced to this model, the "minimal model", a series of other features intended to explore its response under more realistic market conditions, such as the influence of random events. We considered the influence of randomness in the trader's drift, as due to the presence of shocks from news items, or the influence of related markets. Then we considered the influence of a randomly fluctuating rate of entry of new traders into the market, centered around some average. Finally, we considered the diffusion constant itself to vary randomly. We assumed that the fluctuations from the noise were small, so as to generate small corrections to the minimal model.

In addition, we introduced to the minimal model the influence of "market order traders", those who do not register a visible bid or offer in the market before trading. This led us to a two-fluid model, in which the dynamics is very similar to that of the minimal model, but the invisible traders cannot react with one another, and the bid-offer spread is measured only between the visible traders. We proposed this model for the purpose of investigating scaling laws during a crash, as though this might have its own sort of steady state behavior. We may cause it to crash by introducing a large flux of sellers, and we will see that the bid-offer spread widens out in the expected manner, and it would be interesting to use this model to investigate crossover behavior between crashing and non-crashing regimes.

We then added in a drift term to the trader's random walk, whose novel feature is that it changes with the changes in the trade price. This feature is intended to model momentum-trading, or herd-like behavior in traders, and requires that we consider slightly longer time scales, over which these influences may exert themselves. It causes the model to develop crash-like instabilities, stemming from a transition from diffusion dominated dynamics to drift dominated dynamics. It is interesting to study the scaling laws in the prelude to a crash, to observe how they may signal a market's instability. We propose a series of calculations of this kind. They must, however be done numerically, and we postpone these to a future publication 45]. 
Finally, we examined the possibility of treating the more traditional dealer problem in the context of interdealer broker markets, using the proposal of diffusing bids and offers. We found that it allowed us to use the additional information contained in the limit order book to make a more refined model for the order arrival process, and we illustrated this with a toy model in which the dealer's optimization problem for the bid-offer spread was defined.

In future publications, we shall do computer simulations to investigate the more detailed predictions of these models, and compare them directly with live market data 35.

There are several other directions in which research might proceed. One possibility is to observe a market's reaction in response to a shock. In our minimal model, this would correspond to the system being suddenly moved out of its ground state, and partially populating the first few excited states. Because the dynamics of this model are of diffusion type, these excitations will fade away exponentially, with characteristic decay rates given by the "energies" of the excited states. By computing these energies, it is possible to predict the decay rate of market excitations. In a future publication we shall attempt to calculate these decay rates, and again compare them with market data.

Another possibility for future research is to refine the minimal model. We may add refinements in several ways. One way might be, following Garman [3], to introduce a rate $\lambda$ at which traders at the point $x$ enter and leave the market, proportional to their population at $x$. Another way to refine the model would be to introduce interactions between the buyers and sellers. The most obvious kind are those which allow traders to react to what they see on the trading screen. We will discuss in a future publication the "competitive market". This is a market in which traders are anxious to do deals quickly, and as such desire to be the best bid or offer. Bidders are therefore attracted to the best bid, and offers attracted to the best offer, so that they might do the deal first. This would most likely tighten market spreads, and change the scaling law exponents.

Finally, it is interesting to note that the minimal model bears many similarities to members of a class of exactly soluble models that have been elucidated by work over the past two decades[46]. We will explore in a future publication the application of this method to the minimal model.

\section{Appendix}




\section{The form of the time evolution operator}

We introduce an operator, $U_{\text {ann }}$, the "annihilation operator", which takes any state which has sellers and buyers at the same point in price space, and returns the same state with any overlapping buyers and sellers deleted pairwise. If in the state no buyers and sellers overlap, the annihilation operator does not change the state.

We also introduce diffusion separately for the buyers and sellers $D_{B}\left(t, t^{\prime}\right), D_{S}\left(t, t^{\prime}\right)$. These are the usual diffusion operators. When acting on any initial state, the result is a solution to the diffusion equation. However, we cannot allow these diffusion operators to act for very long periods, because at any moment they might cause a seller to jump onto a buyer, or vice versa. Therefore, our diffusion-annihilation operator must diffuse for a very short time, then annihilate, then diffuse some more, annihilate again, etc. We alternate diffusing buyers and sellers, and always insert an annihilation operator in between. Thus, our evolution operator must take the form

$$
E v\left(t, t^{\prime}\right)=\lim _{\delta t \longrightarrow 0} \prod_{n=0}^{\left(t^{\prime}-t\right) / \delta t} U_{\mathrm{ann}} D_{S}(t+n \delta t, t+(n+1) \delta t) U_{\mathrm{ann}} D_{B}(t+n \delta t, t+(n+1) \delta t)(7.1)
$$

Sometimes we will additionally insert into our state a buyer or seller that does not diffuse, but moves according to a prescribed motion. Then this trader must be regarded as a different species, with its own separate evolution, which we mix into the evolution operator. Let the operator defining this prescribed motion be $D_{p}^{(\cdot)}\left(t^{\prime}, t\right)$, where $(\cdot)$ may be either $S$ or $B$. We imagine that the annihilation operator has been appropriately adjusted to take into account annihilations between buyers and sellers of all species. Then Ev becomes

$$
\begin{aligned}
E v\left(t, t^{\prime}\right)= & \lim _{\delta t \longrightarrow 0} \prod_{n=0}^{\left(t^{\prime}-t\right) / \delta t} U_{\mathrm{ann}} D_{p}^{S}(t+n \delta t, t+(n+1) \delta t) U_{\mathrm{ann}} D_{p}^{B}(t+n \delta t, t+(n+1) \delta t) \\
& U_{\mathrm{ann}} D_{S}(t+n \delta t, t+(n+1) \delta t) U_{\mathrm{ann}} D_{B}(t+n \delta t, t+(n+1) \delta t)
\end{aligned}
$$

Obviously, we may introduce as many species as required, and the above provides a template for how we must adjust the evolution operators to properly evolve this more complicated system.

The Form of the Diffusion Operators $D_{B}, D_{S}$

Diffusion operators occur commonly in statistical field theory, and their form is well 
known, see, for example, [48]. We give the standard result here, for completeness.

$$
\begin{array}{ll}
D_{B}\left(t, t^{\prime}\right)=\exp \left(H_{\text {Diff }}^{(B)}\left(t-t^{\prime}\right)\right) & H_{\text {Diff }}^{(B)}=\sum_{x} \Psi_{B}^{\dagger}(x+1) \Psi_{B}(x)+\Psi_{B}^{\dagger}(x-1) \Psi_{B}(x) \\
D_{S}\left(t, t^{\prime}\right)=\exp \left(H_{\text {Diff }}^{(S)}\left(t-t^{\prime}\right)\right) & H_{\text {Diff }}^{(S)}=\sum_{x} \Psi_{S}^{\dagger}(x+1) \Psi_{S}(x)+\Psi_{S}^{\dagger}(x-1) \Psi_{S}(x)
\end{array}
$$

\section{The Form of the Annihilation Operator $U_{\text {ann }}$}

The annihilation operator is an operator that, at every point, eliminates pairs of buyers and sellers that sit on top of one another. After the action of $U_{\text {ann }}$ every point contains either buyers only or sellers only.

$U_{\text {ann }}$ can be written as a product of operators $U_{\text {ann }}(x)$ at each point $U_{\text {ann }}=\prod_{x} U_{\text {ann }}(x)$, whose job is to annihilate pairs at the point $x$. A simple operator that accomplishes this is

$$
U_{\text {ann }}(x)=\left(\Psi_{x}^{(B)} \Psi_{x}^{(S)}\right)^{\min \left(N_{x}^{(B)}, N_{x}^{(S)}\right)}
$$

however, this is not particularly analytically tractable. We may develop another form by considering the master equation for chemical reactions at a point $x$, which occur at rate $\lambda$. Let $P\left(n_{b}, n_{s}\right)$ be the probability of having $n_{b}$ buyers and $n_{s}$ sellers at point $x$. Consider a price space with only a single point. Then the reaction probability per unit time is proportional to the number of particles present $\sim \lambda n_{b} n_{s} P\left(n_{b}, n_{s}\right)$, so that

$$
\frac{\partial P\left(n_{b}, n_{s}\right)}{\partial t}=\lambda\left(\left(n_{b}+1\right)\left(n_{s}+1\right) P\left(n_{b}+1, n_{s}+1\right)-n_{b} n_{s} P\left(n_{b}, n_{s}\right)\right)
$$

For a price space with many points, and a state described by a probability functional $P\left(n_{b}, n_{s}\right)$ which is a functional of integer sequences $n_{b}, n_{s}$ on the price space $\mathcal{P}$, this is easily generalized to

$$
\frac{\partial P\left(n_{b}, n_{s}\right)}{\partial t}=\lambda \sum_{x}\left(\left(n_{b}(x)+1\right)\left(n_{s}(x)+1\right) P\left(n_{b}+1, n_{s}+1\right)-n_{b}(x) n_{s}(x) P\left(n_{b}, n_{s}\right)\right)
$$

This may be expressed in terms of the action of the field operators $\Psi_{B}(x), \Psi_{S}(x), \Psi_{B}^{\dagger}(x), \Psi_{S}^{\dagger}(x)$, as

$$
\frac{\partial P\left(n_{b}, n_{s}\right)}{\partial t}=H_{\mathrm{ann}} P\left(n_{b}, n_{s}\right)
$$


where $H_{\text {ann }}$ is

$$
H_{\mathrm{ann}}=\sum_{x} H_{\mathrm{ann}}(x) \quad H_{\mathrm{ann}}(x)=\lambda\left(\Psi_{B}^{\dagger}(x) \Psi_{S}^{\dagger}(x)-1\right) \Psi_{B}(x) \Psi_{S}(x)
$$

so that $U_{\text {ann }}(x)=\exp \left(H_{\text {ann }}(x) \Delta t\right)$, where $\Delta t$ is the time interval over which this operator is allowed to act.

In general, an annihilation operator of this form does not completely annihilate the particles we want annihilated, but the population of such particles decays exponentially, at the rate $\lambda$. Because of this, sellers can sometimes overlap buyers, a feature which is unrealistic. However, by taking the rate $\lambda \Delta t$ to be very large, this effect can be made arbitrarily small.

The reader should note that this annihilation operator annihilates buyers of a specific type with sellers of a specific type. When there is more than one type, and consequently more than one reaction pair,

then we must introduce an annihilation operator for each pair, and the full annihilation operator is the product of all of them.

Fields with Memory: The Instantaneous Deal Rate Operator, and the Last Trade Field

On a trading screen, one often sees the price at which the most recent trade occured, regardless of how long ago it may have taken place. We may also infer from a trading screen the rate at which trades occur, on average, the "deal rate". We demonstrate how these and related quantities, that require some memory in the system, may be calculated within this kind of model. It will prove convenient to compute these in terms of an operator $N_{\zeta}(x, t)$, which, when acting on a pure state at time $t$ increases by 1 at point $x$ if a trade has occurred at time $t$ and price $x$, and zero otherwise. The instantaneous deal ra te $J(t)$ is then $J(t)=\sum_{x}^{\prime} N_{\zeta}\left(x^{\prime}, t\right)$, and the cumulative deal rate $J_{\text {cum }}(t)$ is $J_{\text {cum }}(t)=\int^{t} d t^{\prime} \sum_{x}^{\prime} N_{\zeta}\left(x^{\prime}, t^{\prime}\right)$. $J_{\text {cum }}(t)$ is a nondecreasing function of $t$, and as such we may define an inverse mapping $J_{\text {cum }}^{-1}(t)$, which associates to a total deal volume the first time it was achieved.

The last trade field $\chi(x, t)$ is constructed as follows. Consider $\tilde{\chi}(t)=\sum_{x}^{\prime} x^{\prime}(d / d t) N_{\zeta}\left(x^{\prime}, t\right)$. If trades occur at one and only one trade price at every time slice $t$ (as they must), then $\tilde{\chi}(t)$ is equal to, at time $\mathrm{t}, x$ if a trade occured at time $t$, and 0 otherwise. This is unsatisfactory for the last trade field, because we want to know the price at which the last trade occurred, regardless of how long ago it occurred. $\tilde{\chi}(t)$ may be thought of as the "non-persistent" last trade field. The "persistent" trade field $\chi(t)$, the one we are after, is then related to the nonpersistent trade field $\tilde{\chi}(t)$ by $\chi(t)=\tilde{\chi}\left(J_{\text {cum }}^{-1}\left(J_{\text {cum }}(t)\right)\right)$. 
The operator $N_{\zeta}$ introduced above is the number operator for a new field $\zeta$, with destruction $\zeta_{x}$ and creation operator $\zeta_{x}^{\dagger}, N_{\zeta}(x)=\zeta_{x}^{\dagger} \zeta_{x} / \delta S$. In order that $N_{\zeta}(x)$ satisfy what is required of it, we must insert a $\zeta$ where a trade occurs. To do this, we modify the annihilation operator $U_{\text {ann }}$. The pairwise annihilation factors each become

$$
H_{\mathrm{ann}}(x)=\lambda\left(\Psi_{B}^{\dagger}(x) \Psi_{S}^{\dagger}(x)-1\right) \zeta^{\dagger}(x) \Psi_{B}(x) \Psi_{S}(x)
$$

Note that the field $\zeta$ differs from the others we have introduced, because it does not represent a trader. It is a mathematical device that records trading information in the position of the field $\zeta$.

\section{Acknowledgements.}

It is a pleasure to thank Judith Bender, Jean-Philippe Bouchaud, Frank Brown,John Cardy, Marc Devorsetz, Amy Eliezer, Fabian Essler, Andrew Graham, Ron Kantor, James Lee, Naresh Malhotra, Peter Orland, Zoltan Racz, Magnus Richardson, Sven Sandow, Fred Schmidt, David Sherrington, Robin Stinchcombe and David Vines for useful discussions and interest in this project. We would like to thank John Cardy, Zoltan Racz and Magnus Richardson for elucidating the theory of diffusion-controlled annihilation. We are indebted to David Sherrington for invaluable help during the preparation of the final version of this paper.

\section{References}

[1] M. O'Hara Market Microstructure Theory, Blackwell Publishers, Inc. (1995)

[2] R. A. Schwartz Reshaping the Equity Markets , HarperCollins, Inc. (1991)

[3] M. Garman Market Microstructure, J. Fin. Econ. 3 , 257 (1976)

[4] H. Stoll The Supply of Dealer Services in Securities Market, J. Fin. 331133 (1978) H. Stoll The Pricing of Security Dealer Services: An Empirical Study of NASDAQ Stocks, J. Fin. 331153 (1978)

[5] T. Ho and H. Stoll On Dealer Markets Under Competition, J. Fin 35259 (1980) T. Ho and H. Stoll Optimal Dealer Pricing Under Transactions and Return Uncertainty, J. Fin. Econ. 947 (1981) 
T. Ho and H. Stoll The Dynamics of Dealer Markets Under Competition, J. Fin 38 $1053(1983)$

[6] M. O'Hara and G. Oldfield The Microeconomics of Market Making, J. Fin. Quant. Anal. 21361 (1986)

[7] Y. Amihud and H. Mendelson Trading Mechanisms and Stock Returns: An Empirical Investigation J. Fin. 42533 (1987)

[8] K. Cohen, S. Maier, R. Schwartz, and D. Whitcomb Transaction Costs, Order Placement Strategy, and Existence of Bid-Offer Spread, J. Pol. Econ. 89287 (1981)

[9] Bagehot, W., [pseud.] The Only Game in Town Fin. An. J. 27, 12-14, (1971) Copeland, T. and Galai, D. Information Effects and the Bid-Ask Spread, J. Fin. 38 $1457(1983)$

Glosten, L. and Milgrom, P. Bid, Ask and Transaction Prices in a Specialist Market with Heterogeneously Informed Traders, J. Fin. Econ. 1371 (1985)

Easley, D. and O'Hara, M. Price, Trade Size, and Information in Securities Markets, J. Fin. Econ. 1969 (1987)

Milgrom, P. and Stokey, N. Information, Trade, and Common Knowledge, J. Econ. Theory 2617 (1982)

Mas-Colell, A. Noncooperative Approaches to the Theory of Perfect Competition: Presentation, J. Econ. Theory 221 (1980)

Black, F. Noise, J. Fin. 43540 (1989)

Roll, R. A Simple Implicit Measure of the Effective Bid-Ask Spread in an Efficient Market, J. Fin. 391127 (1984)

[10] Back, K. Insider Trading in Continuous Time, Rev. Fin. Stud. 5387 (1992) Blume, L. E. and Easley, D. Evolution and Market Behavior, J. Econ. Theory, 58207 (1990) Grossman, S. J. and Stiglitz, J. E. On the Impossibility of Informationally Efficient Markets, Am. Econ. Rev. 70393 (1980)

Kyle, A. S. Market Structure, Information, Futures Markets and Price Formation, in International Agricultural Trade: Advanced Readings in Price Formation, Market Structure, Price Instability, ed. by G. Story, A. Schmitz, and A. Sarris (Westview Press) (1984)

Kyle A. S. Continuous Auctions and Insider Trading, Econometrica 531315 (1985) 
Foster, F. and Viswanathan, S. Variations in Trading Volume, Return Volatility and Trading Costs: Evidence on Recent Price Formation Models, J. Fin. 48, 187 (1993) Holden, C. W. and Subrahmanyam, A. Long-Lived Private Information and Imperfect Competition, J. Fin. 47247 (1992)

Subrahmanyam, A. Risk Aversion, Market Liquidity, and Price Efficiency, 4417 (1991)

Rochet, J. C. and Vila, J. L. Insider Trading and Market Manipulations: Existence and Uniqueness of Equilibrium, Rev. Econ. Stud. (1993)

Jackson, M. Equilibrium, Price Formation, and the Value of Private Information Rev. Fin. Stud. 41 (1991)

Jain, P. C.and Joh, G.-H. The Dependence Between Hourly Prices and Trading Volume, J. Fin. and Quant. Anal. 23336 (1988)

French, K. R.and Roll, R. Stock Return Variances: The Arrival of Information and the Reaction of Traders, J. Fin. Econ. 175 (1986)

Admati, A., and Pfleiderer, P. A Theory of Intraday Patterns: Volume and Price Variability, Rev. Fin. Stud. 13 (1988)

Admati, A., and Pfleiderer, P. Divide and Conquer: A Theory of Intraday and Dayof-the-Week Mean Effects, Rev. Fin. Stud. 2189 (1989)

Seppi, D. Block Trading and Information Revelation Around Quarterly Earning Announcements, Rev. Fin. Stud. 5281 (1990)

Spiegel, M. and Subrahmanyam, A. Informed Speculation and Hedging in an Noncompetitive Securities Market, 5307 (1992)

[11] K. Cohen, S. Maier, R. Schwartz, and D. Whitcomb The Returns Generation Process, Returns Variance, and the Effect of Thinness in Securities Markets, J. Fin. 33, 149 (1978)

[12] K. Cohen, G. Hawawini, S. Maier, R. Schwartz, and D. Whitcomb Implications of Microstructure Theory for Empirical Research on Stock Price Behavior, J. Fin. 35, $249(1980)$

[13] H. Demsetz, The Cost of Transacting, Q. J. Econ. 82 33, (1968)

[14] Tinic, S. M. The Economics of Liquidity Services, Quant. J. Econ. 8679 (1972)

[15] Tinic, S. M. and West, R. R. Competition and the Pricing of Dealer Service in the Over-the-Counter Market, J. Fin. and Quant. Anal. 71707 (1972) 
[16] Tinic, S. M. and West, R. R. Marketability of Common Stocks in Canada and the USA: A Comparison of Agent versus Dealer Dominated Markets, J. Fin. 29729 (1974)

[17] Smidt, S. Continous vs. Intermittent Trading on Auction Markets, Cornell U. (1979)

[18] Schleef, H. and Mildenstein, E. A Dynamic Model of the Security Dealer's Bid and Ask Prices, Proc. Western Econ. Assoc.(Las Vegas) (1979)

[19] Newton, W. and Quandt, R. An Empirical Study of Spreads, Princeton U. (1979)

[20] Hamilton, J. Marketplace Organization and Marketability: NASDAQ, the Stock Exchange, and the National Market System, J. Fin. 33487 (1978)

[21] Hamilton, J. Competition, Scale Economies, and Transaction Cost in the Stock Market, J. Fin. and Quant Anal. 11779 (1976)

[22] Stoll, H. The Pricing of Security Dealer Services: Study of NASDAQ Stocks, J. Fin. 331153 (1978)

[23] Benston G. and Hagerman, R. Determinants of Bid-Asked Spreads in the Over-theCounter Market, J. Fin. Econ. 1353 (1974)

[24] K. Cohen, S. Maier, R. Schwartz, and D. Whitcomb Market Makers and the Market Spread: A Review of Recent Literature, J. Fin. and Quant. Anal. 14813 (1979)

[25] Branch. B, and Freed, W. Bid-Asked Spreads on the Amex and the Big Board, J. Fin. 32159 (1977)

[26] Oldfield, G., Rogalski, R. and Jarrow, R. An Autoregressive Jump Process for Common Stock Returns, J. Fin. Econ. 5389 (1977)

[27] P.Bak, M. Paczuski and M.Shubik, Price Variations in a Stock Market With Many Agents, Physica A 246, 430 (1997), cond-mat/9609144

[28] L.Gálfi and Z.Rácz, Phys. Rev. A 38, 3151 (1988).

[29] B.P. Lee and J.L. Cardy, Phys. Rev. E 50, R3287 (1994);

M.Howard and J.L. Cardy, J.Phys. A 28, 3599 (1995).

G.T. Barkema, M.J. Howard and J.L. Cardy, Phys. Rev. E 53, R2017 (1996). 
[30] S. Cornell and M.Droz, Phys. Rev. Lett. 70, 3824 (1993);

M.Araujo, H. Larralde, S. Halvin and H.E. Stanley, Phys. Rev. Lett. 71, 3592 (1993); Phys. Rev. Lett. 75, 2251 (1995);

S. Cornell, Phys. Rev. Lett. 75, 2250 (1995); Phys. Rev. E 51, 4055 (1995);

[31] M. Doi, J.Phys. A 9, 1465; 1479 (1976).

[32] L.Peliti, J. Physique 46, 1469 (1985).

[33] D. C. Mattis and M. L. Glasser, The uses of Quantum Field Theory in DiffusionLimited Reactions, Rev. Mod. Phys. 70, No. 3, 979, (1998)

[34] A.A. Ovchinnikov, S.F. Timashev and A.A. Belyy, Kinetics of Diffusion Controlled Chemical Processes, Nova Science Publishers, New York, (1990).

[35] D. Eliezer and I.I. Kogan, Experimental Tests of Scaling laws for Microstructure in Double Auction Markets., work in progress.

[36] L. Bachelier, Annales Scientifiques de l'Ecole Normale Superieure, III:17, 21 (1900).

[37] F.Black and M.Scholes, Journal of Political Economy, 81, 636 (1973).

[38] B. Mandelbrot, J.Business 36, 394 (1963); 39, 242 (1966);40, 393 (1967).

[39] P.W. Anderson, K.J. Arrow and D. Pines (eds) The Economy as an evolving complex system, Addison-Wesley (1988).

[40] see for example web cite http://www.eleves.ens.fr:8080/home/cont/ecophysics.html and links there.

[41] R. N. Mantegna and H. E. Stanley, Scaling behaviour in the dynamics of an economic index, Nature, Vol. 376, 46 (1995)

A.Arneodo et al, Comment on "Turbulent cascades in foreign exchange markets", cond-mat/9607120

R. Cont, M. Potters and J.-P.Bouchaud, Scaling in stock market data: stable laws and beyond, Scale Invariance and Beyond (proceedings of the CNRS Workshop on Scale Invariance, Les Houches, March 1997),cond-mat/9705087

[42] D. Sornette, A.Johansen, J.-P.Bouchaud, Stock market crashes, Precursors and Replicas, J.Phys. I France 6, 167 (1996); cond-mat/9510036; 
J.A. Feigenbaum and P.G.O. Freund, Discrete Scaling in Stock Markets Before Crashes, Int. J. Mod. Phys. 10, 3737 (1996),cond-mat/9509033; Discrete Scale Invariance and the "Second Black Monday", cond-mat/9710324

D. Sornette, A.Johansen, Large financial crashes, Physica A245, 411 (1997); condmat/9704127;

A. Johansen, D. Sornette, Stock market crashes are outliers, cond-mat/9712005;

L. Laloux et al, Are Financial Crashes Predictable?, cond-mat/9804111.

[43] J.-P. Bouchaud, N. Sagna, R. Cont, N. El-Karoui, M. Potters, Phenomenology of the Interest Rate Curve, cond-mat/9712164;

D. Sornette, "String" formulation of the Dynamics of the Forward Interest Rate Curve, cond-mat/9802136;

[44] M. Potters, R.Cont and J.-P.Bouchaud, Financial markets as adaptative ecosystems, cond-mat/9609172

J.-P. Bouchaud, R. Cont, A Langevin Approach to Stock Market Fluctuations and Crashes, cond-mat/9801279.

[45] M. Richardson, D. Eliezer and I.I. Kogan, Numerical Simulations of Market Microstructure in Double Auction Markets., work in progress.

[46] Shastry, B. S., Jha S. S. and Singh, V. (eds.) Exactly Solvable Problems in Condensed Matter and Relativistic Field Theory, Springer Verlag, Lecture Notes in Physics 242 (1985);

F. H. L. Essler and V. Rittenberg, J. Phys. A 29, 3375 (1996)

K. Mallick, and S. Sandow, J. Phys. A 30, 4513 (1997);

S. Sandow, Phys. Rev. E 502660 (1994);

L-H.Gwa and H.Spohn, Phys. Rev. A 46, 844 (1992);

[47] C. Itzykson and M. Zuber Quantum Field Theory, McGraw-Hill (1980)

[48] Creutz, M. Quarks, Gluons, and Lattices, Cambridge University Press, (1983) 